\title{
Tractable Fragments of Temporal Sequences of Topological Information
}

\author{
Quentin Cohen-Solal \\ LAMSADE, Université Paris-Dauphine, PSL, CNRS, France \\ quentin.cohen-solal@dauphine.psl.eu
}

\begin{abstract}
In this paper, we focus on qualitative temporal sequences of topological information. We firstly consider the context of topological temporal sequences of length greater than 3 describing the evolution of regions at consecutive time points. We show that there is no Cartesian subclass containing all the basic relations and the universal relation for which the algebraic closure decides satisfiability. However, we identify some tractable subclasses, by giving up the relations containing the non-tangential proper part relation and not containing the tangential proper part relation.

We then formalize an alternative semantics for temporal sequences. We place ourselves in the context of the topological temporal sequences describing the evolution of regions on a partition of time (i.e. an alternation of instants and intervals). In this context, we identify large tractable fragments.
\end{abstract}

Keywords: Qualitative Spatio-temporal Reasoning · Satisfiability Decision.

\section{Introduction}

The reasoning on temporal and spatial qualitative information is necessary to solve many problems that are found in the context of planning, simulation, robotics, intelligent environments and human-computer interaction [28|9|48|13|32|42]. For this reason, many spatio-temporal formalisms have been proposed [23|34|49|43 45 44 8 |31|5| 12 ]. Spatio-temporal formalisms generally decompose into a spatial formalism and a temporal formalism. The point algebra is a formalism describing the relative positions of points on a line (the timeline or a line of space). $\mathrm{RCC}_{8}$ is another formalism, more expressive than the point algebra, expressing the topological relations between regions. It expresses the notions of contact and inclusion.

The qualitative temporal sequences [498] are the simplest spatio-temporal descriptions, in the sense that there is no uncertainty about temporal information. However, strong negative results have been identified for one of the simplest spatial formalisms: deciding the satisfiability of a temporal sequence over the point algebra is NP-complete (even while restricting the language to basic relations and the universal relation) [49]. One can then wonder whether deciding satisfiability is necessarily NP-hard within the framework of spatio-temporal formalisms. However, the complexity of fragments of $\mathrm{RCC}_{8}$ has not been studied within the context of temporal sequences. There could be fragments, not expressing the point algebra, which are tractable. 
We therefore study in this paper the complexity of deciding the satisfiability of the topological temporal sequences. We identify in particular a negative result: the classical procedure to decide the satisfiability of polynomial fragments, the algebraic closure, does not decide the satisfiability in this context (even while being limited to the basic relations and to the universal relation, if the length of the sequence is greater than 3). We also identify a positive result, by considering semantics different from the classical semantics of temporal sequences. More precisely, we no longer consider that temporal sequences describe the evolutions of entities at neighboring instants. We consider instead that they describe the evolutions of entities on a partition of time (i.e. on an alternation of instants and intervals). In the context of this semantics, we identify large tractable fragments.

In the next section, we present related work, the $\mathrm{RCC}_{8}$ formalism and temporal sequences over $\mathrm{RCC}_{8}$. In Section 3 , we introduce our negative result and we identify tractable fragments that do not contain all the basic relations. Finally, in Section 4 , we formalize the alternative semantics of temporal sequences, present the new reasoning operators, and then identify the large tractable fragments.

Note, this paper is an extended version of [6].

\section{Background}

\subsection{Related Work}

Many works deal with spatio-temporal reasoning and its complexity. Tractable fragments have been identified in the context of topological temporal sequences describing the evolution of constant-size regions at non-neighboring instants (regions can satisfy any relations between the instants) [8]. The temporal sequences that we consider, like those of the NP-completeness result of the point algebra, describe regions at neighboring instants. Temporal sequence ordering (at neighboring instants) is an NP-complete problem for several fundamental formalisms, such as $\mathrm{RCC}_{8}$ [45]. Formalisms with a higher temporal expressivity have also been proposed. For example, $\mathrm{RCC}_{8}$ has been combined with Allen's interval algebra [23]. The cardinal direction calculus has also been combined with the Allen's interval algebra [34].

In general, a qualitative spatio-temporal formalism is based on a transition graph, i.e. a graph representing the possible evolutions of basic relations. It can be a neighbourhood graph [17] or a dominance graph [22|21]. In a neighbourhood graph, two relations $b, b^{\prime}$ are neighbour (i.e. adjacent in the graph), if there exists a pair of evolving entities $\left(e, e^{\prime}\right)$ satisfying $b$ at an instant $t$ and $b^{\prime}$ at an instant $t^{\prime}$, and satisfying $b$ or $b^{\prime}$ between $t$ and $t^{\prime}$. In a dominance graph, a relation $b$ dominates another relation $b^{\prime}$ (i.e. there is an arc from $b^{\prime}$ to $b$ in the graph), denoted by $b \vdash b^{\prime}$, if there exists $t, t^{\prime} \in \mathbb{R}$ and a pair of evolving entities $\left(e, e^{\prime}\right)$ satisfying $b$ at $t$ and satisfying $b^{\prime}$ at each instant of $\left.] t, t^{\prime}\right]$. Many transition graphs have been determined [41|53|47|30|17||15|16|14|54|18|10|37|35|27|37|34].

Spatio-temporal qualitative reasoning is also studied in the context of logics (see [24|25|2|33|44|20|1|51|52|19|4|3]). Deciding the satisfiability of these logics is generally PSPACE-hard. Ontologies of time based on points or/and intervals have been studied [46]. There is, in particular, the Event Calculus, a logic of action and change, which can express properties at instant and interval [26]. 


\subsection{Region Connection Calculus $\mathrm{RCC}_{8}$}

$\mathrm{RCC}_{8}[36$ 29]31] is a classical qualitative formalism [31|5|12]. Thus, it is a triplet $(\mathcal{A}, \mathcal{U}, \varphi)$ where $\mathcal{A}$ is a set of relations forming a finite non-associative binary relation algebra, $\mathcal{U}$ is the universe, i.e. the set of considered entities, and $\varphi$ is a particular interpretation function associating with each relation of $\mathcal{A}$ a relation over $\mathcal{U}$. We denote by $\mathrm{RCA}_{8}$ the algebra of $\mathrm{RCC}_{8}$. The universe $\mathcal{U}$ of $\mathrm{RCC}_{8}$ is the set of regions of a certain topological space $\mathfrak{T}$ (i.e. the non-empty, closed and regular subsets of $\mathfrak{T}$ ). $\mathfrak{T}$ is generally $\mathbb{R}^{n}$. Any algebra $\mathcal{A}$ has special relations, called basic relations. Every relation of $\mathcal{A}$ is a union of basic relations. The 8 basic relations of $\mathrm{RCC}_{8}$ : $\mathrm{DC}$ (disconnected), EC (externally connected), PO (partially overlapping), EQ (equal), TPP (tangential proper part), NTPP (non-tangential proper part), and the converse of the two previous relations are described in Figure 1 and defined in Table 1 . We denote by $\mathcal{B}_{\mathrm{RCC}_{8}}$ the set of the basic relations of $\mathrm{RCC}_{8}$. We denote by $\mathcal{B}_{\mathrm{RCC}_{8}}$ the universal relation (i.e. the union of all relations) and by $\varnothing$ the empty relation. Any algebra $\mathcal{A}$ has several operators: the union $\cup$, the intersection $\cap$, the converse ${ }^{-}$, and the (abstract) composition $\circ$. These operators are used to infer new relations: $x r y \Longrightarrow y \bar{r} x$, $x r y \wedge x r^{\prime} y \Longrightarrow x\left(r \cap r^{\prime}\right) y$, and $x r y \wedge y r^{\prime} z \Longrightarrow x\left(r \circ r^{\prime}\right) z$ (with $r, r^{\prime} \in \mathcal{A}$ and $x, y, z$ being entity variables). The abstract composition $\circ$ of $\mathrm{RCC}_{8}$ is the weak composition: $r \circ r^{\prime}=\bigcup\left\{b \in \mathcal{B}_{\mathrm{RCC}_{8}} \mid \varphi(b) \cap\left(\varphi(r) \circ \varphi\left(r^{\prime}\right)\right) \neq \varnothing\right\}$ with $r, r^{\prime} \in \mathcal{A}$. For example, the composition of relations TPP $\cup$ EQ and TPP is the relation TPP. The composition of basic relations is described in a so-called composition table [29].

\begin{tabular}{|c|c|}
\hline Relation & Definition \\
\hline$\overline{x \mathrm{DC} y}$ & $\neg(x \mathrm{C} y)$ \\
\hline$x \mathrm{P} y$ & $\forall z \quad z \mathrm{C} x \Longrightarrow z \mathrm{C} y$ \\
\hline$x$ PP $y$ & $x \mathrm{P} y \wedge \neg(y \mathrm{P} x)$ \\
\hline$x$ EQ $y$ & $x \mathrm{P} y \wedge y \mathrm{P} x$ \\
\hline$x \mathrm{O} y$ & $\exists z \quad z \mathrm{P} x \wedge z \mathrm{P} y$ \\
\hline$x \mathrm{PO} y$ & $x \mathrm{O} y \wedge \neg(x \mathrm{P} y) \wedge \neg(y \mathrm{P} x)$ \\
\hline$x$ EC $y$ & $x \mathrm{C} y \wedge \neg(x \mathrm{O} y)$ \\
\hline$x$ TPP $y$ & $x \mathrm{PP} y \wedge(\exists z z \mathrm{EC} x \wedge z \mathrm{EC} y)$ \\
\hline$x$ NTPP $y$ & $x$ PP $y \wedge \neg(\exists z z$ EC $x \wedge z$ EC $y)$ \\
\hline$x \overline{\mathrm{TPP}} y$ & $y \operatorname{TPP} x$ \\
\hline$x \overline{\text { NTPP }} y$ & $y \operatorname{NTPP} x$ \\
\hline
\end{tabular}

Table 1. Definitions of RCC relations $(C(x, y)$ is the contact relation, it means that the closedregions $x$ and $y$ intersect ; $P$ is the part relation ; $\mathrm{O}$ the overlap relation ; variables $x, y, z$ are closed regions).

Generally, a description based on $\mathrm{RCC}_{8}$ is a qualitative constraint network, i.e. a conjunction of relations between different entities. For instance, $x$ DC $\cup$ EC $y \wedge$ $z \mathrm{TPP} \cup \mathrm{NTPP} \cup \mathrm{EQ} y$ is such a description, which means that the interiors of regions $x$ and $y$ are disjoint and that the region $z$ is included in $y$. Deciding the satisfiability of qualitative constraint networks whose relations belong to $\mathrm{RCA}_{8}$ is an NP- 


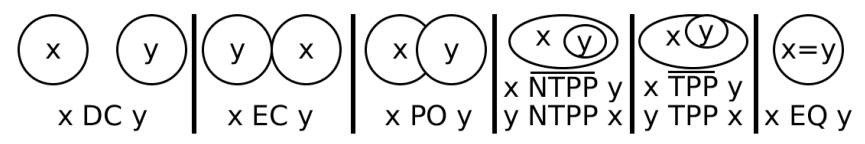

Fig. 1. The 8 basic relations of $\mathrm{RCC}_{8}$ in the plane.

complete problem [38]. Tractable fragments have been identified. They consist in restricting the relations of constraint networks to a particular subset of $\mathrm{RCA}_{8}$. Three large tractable subsets containing all the basic relations and the universal relation have been identified: $\mathcal{H}_{8}, \mathcal{Q}_{8}$, and $\mathcal{C}_{8}$ [38]. They are moreover maximal for tractability. They are defined in Table 2. On these subclasses, applying the algebraic closure, which is a reasoning operator on networks using the algebra operators, decides satisfiability.

\begin{tabular}{|c|c|}
\hline & Definition \\
\hline \hline $\mathcal{N}$ & $\left\{r \in \mathrm{RCA}_{8} \mid \mathrm{PO} \nsubseteq r \wedge r \cap(\mathrm{TPP} \cup \mathrm{NTPP}) \neq \varnothing \wedge r \cap(\overline{\mathrm{TPP}} \cup \overline{\mathrm{NTPP}}) \neq \varnothing\right\}$ \\
\hline $\mathcal{N} \mathcal{P}_{8}$ & $\mathcal{N} \cup\left\{r_{1} \cup \mathrm{EC} \cup r_{2} \cup \mathrm{EQ} \mid r_{1} \in\{\varnothing, \mathrm{DC}\} \wedge r_{2} \in\{\mathrm{NTPP}, \overline{\mathrm{NTPP}}\}\right\}$ \\
\hline $\mathcal{P}_{8}$ & $\mathrm{RCA}_{8} \backslash \mathcal{N} \mathcal{P}_{8}$ \\
\hline $\mathcal{H}_{8}$ & $\mathcal{P}_{8} \cap\left\{r \in \mathrm{RCA}_{8} \mid \mathrm{NTPP} \cup \mathrm{EQ} \subseteq r \Longrightarrow \overline{\mathrm{TPP}} \subseteq r \wedge \overline{\mathrm{NTP}} \cup \mathrm{EQ} \subseteq r \Longrightarrow \overline{\mathrm{TPP}} \subseteq r\right\}$ \\
\hline $\mathcal{Q}_{8}$ & $\mathcal{P}_{8} \cap\left\{r \in \mathrm{RCA}_{8} \mid(\mathrm{EQ} \subseteq r \wedge r \cap(\mathrm{TPP} \cup \mathrm{NTPP} \cup \overline{\mathrm{TPP}} \cup \overline{\mathrm{NTPP}}) \neq \varnothing) \Longrightarrow \mathrm{PO} \subseteq r\right\}$ \\
\hline $\mathcal{C}_{8}$ & $\mathcal{P}_{8} \cap\left\{r \in \mathrm{RCA} A_{8} \mid(\mathrm{EC} \subseteq r \wedge r \cap(\mathrm{TPP} \cup \mathrm{NTPP} \cup \overline{\mathrm{TPP}} \cup \overline{\mathrm{NTPP}} \cup \mathrm{EQ}) \neq \varnothing) \Longrightarrow \mathrm{PO} \subseteq r\right\}$ \\
\hline
\end{tabular}

Table 2. Definitions of the relations sets $\mathcal{H}_{8}, \mathcal{Q}_{8}$, and $\mathcal{C}_{8}$.

\subsection{Link with finite CSP}

We briefly discuss the links between qualitative formalisms and finite CSP. On the one hand, for some qualitative formalisms, the algebraic closure enforces path-consistency [39]. On the other hand, a qualitative constraints network can be translated into a network of finite quantitative constraints [50]. The CSP variables are the relations between the qualitative variables. More precisely, there is a CSP variable $v_{x y}$ for each pair of qualitative variables $(x, y)$. The set of possible values for the CSP variable $v_{x y}$ is the set of basic relations contained in the relation between $x$ and $y$. The CSP constraints between the CSP variables encode the composition operator. These constraints are ternary and of the form $\left\{\left(b^{\prime \prime}, b, b^{\prime}\right) \in \mathcal{B}^{3} \mid b^{\prime \prime} \subseteq b \circ b^{\prime}\right\}$.

\subsection{Semantics of continuously evolving regions}

Before presenting temporal sequences over $\mathrm{RCC}_{8}$, we must formally define what we call a region evolving continuously over time. A region evolving continuously during a time interval $I$ (i.e. a real closed interval) is naturally defined as a continuous function $f$ from $I$ to the set of considered regions $\mathfrak{R}$ of a topological space (for instance, $\mathfrak{R}$ can be the regions of $\mathbb{R}^{n}$ with $n \geq 1$ and can possibly be restricted to convex or connected 
regions). However, this standard mathematical definition requires that $\mathfrak{R}$ be associated with a topology. Thus, we require the following concept:

Definition 1. A topological region space $(\mathfrak{R}, T)$ is a set of regions $\mathfrak{R}$ of a topological space associated with a topology $T$ (i.e. $(\mathfrak{R}, T)$ is also a topological space).

There are several possible topologies for the regions of $\mathbb{R}^{n}$ [21|11]. In particular, choosing a metric between regions amounts to choosing a topology. Depending on the choice of the topological region space, the evolution of regions satisfies or violates certain properties, such as continuity of particular functions (area, distance, union, projection, convex hull, ...) [11]. In fact, solids, gases, shadows, ... do not evolve continuously in the same way [21]. The usual metric of the regions of $\mathbb{R}^{n}$ is the Hausdorff distance. Unfortunately, the corresponding evolution of the relations of regions is not compatible with the classical neighbourhood graph of $\mathrm{RCC}_{8}$ (Figure 2] a) [11]. The dual-Hausdorff distance [11] corrects this problem: the evolution of regions according to this metric is compatible with the classical neighbourhood graph of $\mathrm{RCC}_{8}$. Note that other metrics also correct it.

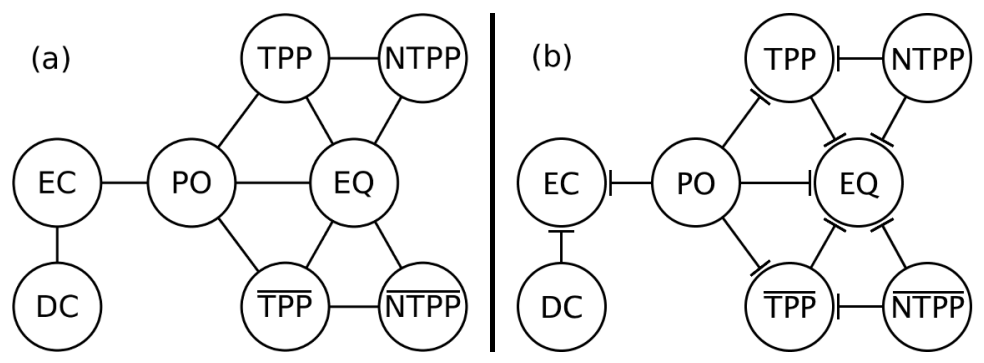

Fig. 2. Neighbourhood graph of $\mathrm{RCC}_{8}$ (a) and dominance graph of $\mathrm{RCC}_{8}$ (b).

\subsection{Topological Sequences at Neighboring Instants}

We present in this section the topological temporal sequences describing the continuous evolution of regions at neighboring instants [8], that we denote $\mathrm{TRCC}_{8}^{\mathrm{n}}$. For this, we recall the basics of the framework of multi-algebras [7|8] from which it is defined. It is an abstract framework that includes several extensions of classical qualitative formalisms, such as temporal sequences.

Projections and Relations Multi-algebras generalize non-associative binary relation algebras. A multi-algebra is a Cartesian product $\mathcal{A}=\mathcal{A}_{1} \times \cdots \times \mathcal{A}_{m}$ of relation algebras satisfying certain properties. We denote by $I$ the index set of the multi-algebra, i.e. $\{1, \ldots, m\}$. In the context of temporal sequences, each algebra $\mathcal{A}_{i}$ corresponds to the same relation algebra but to a different time period. The set of basic relations of $\mathcal{A}$, denoted $\mathcal{B}$, is $\mathcal{B}_{1} \times \cdots \times \mathcal{B}_{m}$ where $\mathcal{B}_{i}$ is the set of basic relations of $\mathcal{A}_{i}$. Multi-algebras 
are equipped with a set of additional operators $\Gamma_{i}^{j}$ from $\mathcal{A}_{i}$ to $\mathcal{A}_{j}$ for all distinct $i, j \in \mathrm{I}$, called projections. Any projection $\gg$ satisfies by definition $\gg\left(r \cup r^{\prime}\right)=(\risingdotseq r) \cup\left(\risingdotseq r^{\prime}\right)$ and $\Gamma(\bar{r})=\bar{\longmapsto}(r)$. In the context of temporal sequences, projections describe the possible evolution of relations over time.

\begin{tabular}{|c|c|}
\hline$b$ & $\uparrow b$ \\
\hline \hline $\mathrm{DC}$ & $\mathrm{DC} \cup \mathrm{EC}$ \\
\hline $\mathrm{EC}$ & $\mathrm{DC} \cup \mathrm{EC} \cup \mathrm{PO}$ \\
\hline $\mathrm{PO}$ & $\mathrm{EC} \cup \mathrm{PO} \cup \mathrm{TPP} \cup \overline{\mathrm{TPP}} \cup \mathrm{EQ}$ \\
\hline $\mathrm{TPP}$ & $\mathrm{PO} \cup \mathrm{TPP} \cup \mathrm{NTPP} \cup \mathrm{EQ}$ \\
\hline $\mathrm{NTPP}$ & $\mathrm{TPP} \cup \mathrm{NTPP} \cup \mathrm{EQ}$ \\
\hline $\mathrm{EQ}$ & $\mathrm{PO} \cup \mathrm{TPP} \cup \mathrm{NTPP} \cup \overline{\mathrm{TPP}} \cup \overline{\mathrm{NTPP}} \cup \mathrm{EQ}$ \\
\hline
\end{tabular}

Table 3. Neighboring relations of the basic relations of $\mathrm{RCC}_{8}$.

Definition 2. The operator $\uparrow$ from $\mathrm{RCA}_{8}$ to $\mathrm{RCA}_{8}$ is the projection satisfying the Table 3

The projection $\uparrow$ encodes the neighbourhood graph of $\mathrm{RCC}_{8}$ described in Figure $2 \mathrm{a}$ (i.e. $b \subseteq \uparrow b^{\prime}$ if and only if $b$ and $b^{\prime}$ are neighbours). For instance, PO $\nsubseteq \uparrow \mathrm{DC}$ because it is not possible to have a continuous transition from DC to PO. A relation of a multi-algebra is an $m$-tuplet of classical relations. By adding semantics, that is to say a universe $\mathcal{U}$ and a specific interpretation function $\varphi$, we get a qualitative formalism said loosely combined, also called sequential formalism.

Example 1. To illustrate the preceding concepts and to give intuition concerning $\mathrm{TRCC}_{8}^{\mathrm{n}}$, we give some examples ( $\mathrm{TRCC}_{8}^{\mathrm{n}}$ will be formalized in the next subsection). The Cartesian product of the multi-algebra $\mathcal{A}$ of $\mathrm{TRCC}_{8}^{\mathrm{n}}$ is $\mathrm{RCA}_{8}^{m}$ where $m$ is the length of considered temporal sequences (the sequences describe regions at instants $t_{1}, \ldots, t_{m}$ ). The component $i \in \mathrm{I}$ of a relation $R$ of $\mathrm{TRCC}_{8}^{\mathrm{n}}, R_{i}$, is the relation of $\mathrm{RCC}_{8}$ which must be satisfied at the instant $t_{i}$. An example of relations of $\operatorname{TRCC}_{8}^{\mathrm{n}}$, with $m=3$, is $(\mathrm{TPP} \cup \mathrm{NTPP} \cup \overline{\mathrm{TPP}} \cup \overline{\mathrm{NTPP}}, \mathrm{PO} \cup \mathrm{EQ}, \mathrm{EC} \cup \mathrm{DC})$. This relation means on the one hand that one of the two regions is first included in the other $\left(R_{1}\right.$ is satisfied at $\left.t_{1}\right)$, then they overlap or are equal $\left(R_{2}\right.$ is satisfied at $\left.t_{2}\right)$ and finally they are disjoint $\left(R_{3}\right.$ is satisfied at $\left.t_{3}\right)$. Since the instants of the sequence are neighbors, this relation means, on the other hand, that between $t_{1}$ and $t_{2}$ the regions satisfy either the basic relation being satisfied at $t_{1}$ (which is TPP or NTPP or TPP or NTPP) or the one being satisfied at $t_{2}$ (which is PO or EQ) and between $t_{2}$ and $t_{3}$ they satisfy either the basic relation being satisfied at $t_{2}$ (which is $\mathrm{PO}$ or $\mathrm{EQ}$ ) or the one being satisfied at $t_{3}$ (which is EC or DC). This additional constraint is called continuity without intermediary relation and also continuous qualitative change [8 [49]. This constraint enforces that each sequence of relations $R$ describes all changes of relations between regions. In other words, between two instants $t_{i}$ and $t_{i+1}$, there must be no change of relation, other than the transition from the basic relation satisfied at $t_{i}$ towards the basic relation satisfied at $t_{i+1}$. 
Multi-algebra and Relation Operators We recall the definition of $\mathrm{TRCC}_{8}^{\mathrm{n}}$ (originally denoted $\mathrm{TT}_{\text {wir }}[8]$ ).

Definition 3. Let $t_{1}, \ldots, t_{m} \in \mathbb{R}$ be consecutive instants and $(\Re, T)$ be a topological region space. $\operatorname{TRCC}_{8}^{\mathrm{n}}$ is the triplet $(\mathcal{A}, \mathcal{U}, \varphi)$ where:

- $\mathcal{A}$ is $\mathrm{RCA}_{8}^{m}$ equipped with the projections $\Gamma_{i}^{j}$ fully defined by $\Gamma_{i}^{j} b=\uparrow b$ if $|j-i|=$ 1 and $\Gamma_{i}^{j} b=\mathcal{B}_{\mathrm{RCC}_{8}}$ otherwise, for all $b \in \mathcal{B}_{\mathrm{RCC}_{8}}$ and $i, j \in \mathrm{I}$,

$-\mathcal{U}$ is the set of continuous functions from $\left[t_{1}, t_{m}\right]$ to $\mathfrak{R}$, and

- $\varphi$ is the function from $\mathcal{A}$ to $2^{\mathcal{U} \times \mathcal{U}}$ such that for all $R \in \mathcal{A}, \varphi(R)$ is the set of pairs of functions $\left(f, f^{\prime}\right) \in \mathcal{U} \times \mathcal{U}$ satisfying at each instant $t_{i}$ the relation $R_{i}$ (i.e. $\left.\forall i \in \mathrm{I}\left(f\left(t_{i}\right), f^{\prime}\left(t_{i}\right)\right) \in \varphi_{\mathrm{RCC}_{8}}\left(R_{i}\right)\right)$ and satisfying no intermediary relations between each instants $t_{i}$ and $t_{i+1}$ (i.e. during each $\left[t_{i}, t_{i+1}\right]$ a basic relation is satisfied, then another, formally: for all $i \in I \backslash\{m\}$ there exist $\tau \in\left[t_{i}, t_{i+1}\right]$ and $b, b^{\prime} \in \mathcal{B}_{\mathrm{RCC}_{8}}$ such that at each instant $t \in\left[t_{i}, \tau\left[,\left(f(t), f^{\prime}(t)\right) \in \varphi_{\mathrm{RCC}_{8}}(b)\right.\right.$, at each instant $\left.t \in] \tau, t_{i+1}\right],\left(f(t), f^{\prime}(t)\right) \in \varphi_{\mathrm{RCC}_{8}}\left(b^{\prime}\right)$, and that $\left(f(\tau), f^{\prime}(\tau)\right) \in$ $\left.\varphi_{\mathrm{RCC}_{8}}\left(b \cup b^{\prime}\right)\right)$, with $\varphi_{\mathrm{RCC}_{8}}$ the interpretation function of $\mathrm{RCC}_{8}$.

Remark 1. $\mathrm{TRCC}_{8}^{\mathrm{n}}$ depends on a set of regions but also on a topology for the regions (i.e. a notion of continuity). Note that $\mathrm{TRCC}_{8}^{\mathrm{n}}$ is not necessarily a sequential formalism (i.e. its reasoning operators are not necessarily correct: reasoning operators could remove some solutions). To be a sequential formalism, the evolution of regions corresponding to the chosen topological region space must be compatible with the classical neighbourhood graph of $\mathrm{RCC}_{8}$ (see Section 2.4). Thus, if $\mathfrak{R}$ is $\mathbb{R}^{n}$ equipped with the dual-Hausdorff distance, TRCC $_{8}^{\mathrm{n}}$ is a sequential formalism.

Every multi-algebra has operators on its relations, namely composition, union, intersection, and converse. They are defined componentwise. For example, the composition of $R$ and $R^{\prime}, R \circ R^{\prime}$, is defined by $\left(R \circ R^{\prime}\right)_{i}=R_{i} \circ R_{i}^{\prime}$ for all $i \in \mathrm{I}$.

There is another operator on relations: the projection closure of a relation $R$, denoted $\Gamma(R)$. It consists in sequentially applying the following operation until reaching a fixed point: for all $j \in \mathrm{I}, R_{j} \leftarrow R_{j} \cap \bigcap_{i \neq j} \Gamma_{i}^{j} R_{i}$. Projection closure refines relations by removing classical basic relations that are impossible to satisfy. In the context of $\mathrm{TRCC}_{8}^{\mathrm{n}}$, projection closure enforces continuity without intermediary relation. For example, the projection closure of the following relation, with $m=3$, $(\mathrm{TPP} \cup \mathrm{NTPP} \cup \overline{\mathrm{TPP}} \cup \overline{\mathrm{NTPP}}, \mathrm{PO} \cup \mathrm{EQ}, \mathrm{EC} \cup \mathrm{DC})$ is (TPP $\cup \overline{\mathrm{TPP}}, \mathrm{PO}, \mathrm{EC})$. Indeed, in particular, there is no transition from the relation $\mathrm{PO}$ or from the relation EQ to the relation DC without intermediary relation. In addition, there is no transition from NTPP to DC or EC in just two qualitative changes. Projection closure removes such impossibilities. Relations closed under projection, i.e. satisfying $\longmapsto(R)=R$, are said $\risingdotseq$-closed. Note that projection closure can be seen as a kind of arc-consistency.

Constraint Networks and Algebraic Closure A description in the context of multialgebras is a (qualitative constraint) network. A network over a multi-algebra $\mathcal{A}$ is a set of variables $\mathrm{E}$ and a function $N$ associating with each pair of variables $(x, y) \in \mathrm{E}^{2}$ such that $x \neq y$ a relation of $\mathcal{A}$ and satisfying $N(x, y)=\overline{N(y, x)}$ for all distinct 
$x, y \in \mathrm{E}$. A sequence of classical constraint networks is thus represented by a single constraint network whose relations are sequences of relations, i.e. relations of a multialgebra. We denote $N(x, y)$ more succinctly by $N^{x y}$. It is sometimes useful to refer to the "subnetwork" corresponding to the index $i \in \mathrm{I}$ of a network $N$, denoted $N_{i}$, called slice. $N_{i}$ is defined by $\left(N_{i}\right)^{x y}=\left(N^{x y}\right)_{i}$ for all distinct $x, y \in \mathrm{E}$. In the context of temporal sequences, the slice $i$ of a network $N, N_{i}$, describes the relations of the sequence at the instant $t_{i}$. Similarly, the slice $i \in \mathrm{I}$ of a subset $\mathcal{S} \subseteq \mathcal{A}$, denoted $\mathcal{S}_{i}$, is $\left\{R_{i} \mid R \in \mathcal{S}\right\}$. A network is said to be satisfiable (or consistent) if there is a solution to this network, that is, an assignment for the variables $\left\{u_{x}\right\}_{x \in \mathrm{E}} \subseteq \mathcal{U}$ satisfying the relations of the constraint network, i.e. $\left(u_{x}, u_{y}\right) \in \varphi\left(N^{x y}\right)$. A network $N$ is said over a subset of relations $\mathcal{S} \subseteq \mathcal{A}$ if for all distinct $x, y \in \mathrm{E}, N^{x y} \in \mathcal{S}$. A scenario is a network over $\mathcal{B}$.

The reasoning operator on networks is the algebraic closure, which applies the operators of the multi-algebra. It propagates information within the network, makes inferences, by refining relations. In the context of topological temporal sequences, the algebraic closure propagates information over regions at each instant and between the different instants. A relation $R$ refines a relation $R^{\prime}$ if $R_{i} \subseteq R_{i}^{\prime}$ for all $i \in \mathrm{I}$. More generally, $N$ refines $N^{\prime}$, denoted $N \subseteq N^{\prime}$, if for all distinct $x, y \in \mathrm{E}, N^{x y} \subseteq\left(N^{\prime}\right)^{x y}$. Algebraic closure closes networks under composition and under projection. Algebraic closure thus applies the two following operations until reaching a fixed point: $N^{x z} \leftarrow$ $N^{x z} \cap\left(N^{x y} \circ N^{y z}\right)$ and $N^{x z} \leftarrow \Gamma\left(N^{x z}\right)$ for all distinct $x, y, z \in \mathrm{E}$. We denotes by $\mathfrak{C}(N)$ the algebraic closure of $N$. In the context of topological temporal sequences, the composition operator makes inferences at every instant and the projection operator makes inferences between the instants. A network $N$ is called algebraically closed if it is closed under composition, i.e. for all distinct $x, y, z \in \mathrm{E}, N^{x z} \subseteq N^{x y} \circ N^{y z}$, and if each of its relations $N^{x y}$ is closed under projection, i.e. for all distinct $i, j \in \mathrm{I}$, $N_{j}^{x y} \subseteq \Gamma_{i}^{j} N_{i}^{x y}$.

Consistency and Satisfiability A sequential formalism is said complete if all its algebraically closed scenarios are satisfiable. It is a fondamental property for deciding satisfiability in an algebraic way.

Remark 2. To know if $\mathrm{TRCC}_{8}^{\mathrm{n}}$ is complete for the regions of $\mathbb{R}^{n}$ equipped with the dual-Hausdorff distance or for another topological region space is a complex problem. In fact, perhaps there is no topological region space such that $\mathrm{TRCC}_{8}^{\mathrm{n}}$ is a complete sequential formalism. For this reason, several studies have dealt with a "weak satisfiability", i.e. satisfiability with a weaker notion of continuity [23 49 452]. Formally, a network $N$ over $\mathrm{TRCC}_{8}^{\mathrm{n}}$ is weakly satisfiable if it contains an algebraically closed scenario (i.e. if there exists a sequence of satisfiable classical scenarios satisfying the constraints of the networks $N_{i}$ and the neighbourhood graph). The tractable subclasses that we identify in this article are tractable for $\mathrm{TRCC}_{8}^{\mathrm{n}}$ associated with a topological region space such that $\mathrm{TRCC}_{8}^{\mathrm{n}}$ is a complete sequential formalism. They are also tractable for this notion of weak satisfiability.

A relation $R$ is said trivially unsatisfiable if there exists $i \in \mathrm{I}$ such that $R_{i}=\varnothing$. Note that a relation which is not trivially unsatisfiable can be unsatisfiable, i.e. $\varphi(R)=$ 
$\varnothing$. This is the case of $(\mathrm{PO}, \mathrm{PO}, \mathrm{DC})$. A relation is said $ґ$-consistent if it is $ґ$-closed and it is not trivially unsatisfiable. A network is said trivially unsatisfiable if there exists distinct $x, y \in \mathrm{E}$ such that $N^{x y}$ is a trivially unsatisfiable relation. An algebraically closed network that is not trivially unsatisfiable is said to be algebraically consistent.

Tractable Subclasses By restricting networks to certain subsets of relations $\mathcal{S}$, we get the following property: if the algebraic closure of a network over $\mathcal{S}$ is algebraically consistent, then this network is satisfiable. Such subsets are said to be algebraically tractable. In other words, with an algebraically tractable subset $S$, to decide the satisfiability of a network over $S$, it suffices to verify that its algebraic closure is not trivially inconsistent. The search for algebraically tractable subsets has focused on particular subsets [31]. A subset $\mathcal{S} \subseteq \mathcal{A}$ is said a subclass if it is closed under intersection, composition, and converse (i.e. for all $R, R^{\prime} \in \mathcal{S}$, we have $R \cap R^{\prime} \in \mathcal{S}, R \circ R^{\prime} \in \mathcal{S}$, and $\bar{R} \in \mathcal{S}$ ). Subclasses containing all basic relations (i.e. $\mathcal{B} \subseteq \mathcal{S}$ ) are called subalgebras. A subset $\mathcal{S} \subseteq \mathcal{A}$ is said $ґ$-closed if for all $R \in \mathcal{S}, \Gamma(R) \in \mathcal{S}$. Finally, we say that a subset $\mathcal{S}$ is Cartesian if $\mathcal{S}=\mathcal{S}_{1} \times \cdots \times \mathcal{S}_{m}$.

Note that a list of conditions guaranteeing algebraic tractability has been identified [7] (see the slicing and refinement theorems). One of these conditions is algebraic stability by a refinement $H$. A refinement $H$ is a function from $\mathcal{A}$ to $\mathcal{A}$ satisfying $H(R) \subseteq R$. A subset $\mathcal{S} \subseteq \mathcal{A}$ is algebraically stable by $H$ if for any algebraically consistent network $N$ over $\mathcal{S}$, the network $H(N)$ is algebraically consistent, where $H(N)$ is the network obtained from $N$ by substituting each relation $N^{x y}$ by $H\left(N^{x y}\right) . \mathrm{RCC}_{8}$

has two fundamental refinements: $h_{\mathcal{H}}(r)=\mathrm{a}_{\overline{\mathrm{TPP}}}\left(\mathrm{a}_{\mathrm{TPP}}\left(\mathrm{aPO}_{\mathrm{P} O}\left(\mathrm{a}_{\mathrm{EC}}\left(\mathrm{a}_{\mathrm{DC}}(r)\right)\right)\right)\right)$ and $h_{\mathcal{C}_{8}}(r)=\mathrm{a}_{\overline{\mathrm{TPP}}}\left(\mathrm{a}_{\mathrm{TPP}}\left(\mathrm{a}_{\overline{\mathrm{NTPP}}}\left(\mathrm{a}_{\mathrm{NTPP}}\left(\mathrm{a}_{\mathrm{PO}}\left(\mathrm{a}_{\mathrm{DC}}(r)\right)\right)\right)\right)\right)$ with $\mathrm{a}_{b}, b \in \mathcal{B}$, the function from $\mathrm{RCA}_{8}$ to $\mathrm{RCA}_{8}$ defined by $\mathrm{a}_{b}(r)=b$ if $b \subseteq r$ and $\mathrm{a}_{b}(r)=r$ otherwise. $\mathcal{H}_{8}$ and $\mathcal{Q}_{8}$ are algebraically stable by $h_{\mathcal{H}_{8}}$ and $\mathcal{C}_{8}$ is algebraically stable by $h_{\mathcal{C}_{8}}$ [38]. Moreover, for every $r \in \mathcal{H}_{8} \cup \mathcal{Q}_{8}$ such that $r \neq \varnothing, h_{\mathcal{H}_{8}}(r) \in \mathcal{B}_{\mathrm{RCC}_{8}}$ and for every $r \in \mathcal{C}_{8} \backslash\{\varnothing\}, h_{\mathcal{C}_{8}}(r) \in \mathcal{B}_{\mathrm{RCC}_{8}}$ [38]. In the following, we are interested in the refinement $H_{\mathcal{S}}$ defined by $H_{\mathcal{S}}(R)_{i}=h_{\mathcal{H}_{8}}\left(R_{i}\right)$ if $\mathcal{S}_{i} \subseteq \mathcal{H}_{8}$ or $\mathcal{S}_{i} \subseteq \mathcal{Q}_{8}$ and $H_{\mathcal{S}}(R)_{i}=h_{\mathcal{C}_{8}}\left(R_{i}\right)$ otherwise, for all $i \in \mathrm{I}$ and $R \in \mathrm{RCA}_{8}^{m}$, with $\mathcal{S} \subseteq \mathrm{RCA}_{8}^{m}$.

\section{$3 \quad$ Study of TRCC $_{8}^{\mathrm{n}}$ Subclasses}

In this section, we are interested in temporalized $\mathrm{RCC}_{8}$ at neighboring instants, i.e. $\mathrm{TRCC}_{8}^{\mathrm{n}}$ (see Section 2.5). More precisely, we search for subclasses that are algebraically tractable. Unfortunately, as the following proposition shows, there are no algebraically tractable Cartesian subalgebras (at least for $m \geq 4$ ).

Proposition 1. Let $\mathfrak{R}$ be a topological region space such that $\mathrm{TRCC}_{8}^{\mathrm{n}}$ is a sequential formalism.

No Cartesian subalgebra of $\mathrm{TRCC}_{8}^{\mathrm{n}}$ is algebraically tractable (when $m \geq 4$ ).

No $\risingdotseq$-closed Cartesian subalgebra of $\mathrm{TRCC}_{8}^{\mathrm{n}}$ is algebraically tractable (if $m \geq 2$ ).

Proof. We show the case $m=4$. The idea is that the algebraic closure can produce relations $R$ verifying $\mathrm{TPP} \cup \mathrm{EQ} \subseteq R_{i} \subseteq \mathrm{TPP} \cup \mathrm{NTPP} \cup \mathrm{EQ}$ and $\overline{\mathrm{TPP}} \cup \mathrm{EQ} \subseteq R_{j} \subseteq$ 
$\overline{\mathrm{TPP}} \cup \overline{\mathrm{NTPP}} \cup \mathrm{EQ}$ with $|i-j|=1$ which causes that some unsatisfiable networks are algebraically consistent. Let $\mathcal{S}$ be any Cartesian subalgebra (thus $\mathcal{S}$ contains the closure of the basic relations and the universal relation of $\mathrm{RCC}_{8}$ under intersection, composition, and converse). We show that there exists an unsatisfiable network over $\mathcal{S}$ whose algebraic closure is algebraically consistent. Consider the network $N$ satisfying: $\mathrm{E}=\{u, v, w, x, y, z\}, N_{1}^{x y}=\mathrm{NTPP}, N_{4}^{x y}=\overline{\mathrm{NTPP}}, N_{1}^{x z}=\mathrm{NTPP}, N_{3}^{w z}=\mathrm{NTPP}$, $N_{1}^{y z}=\overline{\mathrm{TPP}}, N_{2}^{y z}=\mathrm{PO} \cup \mathrm{TPP} \cup \overline{\mathrm{TPP}} \cup \mathrm{EQ}, N_{1}^{w x}=\overline{\mathrm{TPP}}, N_{2}^{w x}=\mathrm{PO} \cup \mathrm{TPP} \cup$ $\overline{\mathrm{TPP}} \cup \mathrm{EQ}, N_{2}^{w y}=\mathrm{PO} \cup \mathrm{TPP}, N_{4}^{x u}=\overline{\mathrm{NTPP}}, N_{2}^{v u}=\overline{\mathrm{NTPP}}, N_{4}^{y u}=\mathrm{TPP}$, $N_{3}^{y u}=\mathrm{PO} \cup \mathrm{TPP} \cup \overline{\mathrm{TPP}} \cup \mathrm{EQ}, N_{4}^{v x}=\mathrm{TPP}, N_{3}^{v x}=\mathrm{PO} \cup \mathrm{TPP} \cup \overline{\mathrm{TPP}} \cup \mathrm{EQ}$, $N_{3}^{v y}=\mathrm{PO} \cup \overline{\mathrm{TPP}}$, and $N_{i}^{a b}=\mathcal{B}_{\mathrm{RCC}_{8}}$ in the other cases. The network is over $\mathcal{S}$ (indeed, we have $\mathrm{DC} \circ \mathrm{DC}=\mathcal{B}_{\mathrm{RCC}_{8}}, \overline{\mathrm{TPP}} \circ \mathrm{TPP}=\mathrm{PO} \cup \mathrm{TPP} \cup \overline{\mathrm{TPP}} \cup \mathrm{EQ}$, and $(\mathrm{EC} \circ \mathrm{EC}) \cap(\mathrm{EC} \circ \mathrm{NTPP})=\mathrm{PO} \cup \mathrm{TPP})$. Its algebraic closure $\mathfrak{C}(N)$ is the algebraically consistent network satisfying:

$-\mathfrak{C}(N)^{x y}=(\mathrm{NTPP}, \mathrm{TPP} \cup \mathrm{NTPP} \cup \mathrm{EQ}, \overline{\mathrm{TPP}} \cup \overline{\mathrm{NTPP}} \cup \mathrm{EQ}, \overline{\mathrm{NTPP}})$,

- $\mathfrak{C}(N)^{y z}=(\overline{\mathrm{TPP}}, \mathrm{PO} \cup \overline{\mathrm{TPP}} \cup \mathrm{EQ}, \mathcal{B} \backslash(\mathrm{DC} \cup \mathrm{EC}), \mathcal{B} \backslash \mathrm{DC})$,

$-\mathfrak{C}(N)^{x z}=(\mathrm{NTPP}, \mathrm{TPP} \cup \mathrm{NTPP} \cup \mathrm{EQ}, \mathcal{B} \backslash(\mathrm{DC} \cup \mathrm{EC}), \mathcal{B} \backslash(\mathrm{DC} \cup \mathrm{EC}))$,

$-\mathfrak{C}(N)^{w x}=(\overline{\mathrm{TPP}}, \mathrm{PO} \cup \overline{\mathrm{TPP}} \cup \mathrm{EQ}, \mathcal{B} \backslash(\mathrm{DC} \cup \overline{\mathrm{NTPP}}), \mathcal{B})$,

$-\mathfrak{C}(N)^{w y}=(\mathrm{PO} \cup \mathrm{TPP} \cup \mathrm{NTPP}, \mathrm{PO} \cup \mathrm{TPP}, \mathcal{B} \backslash(\mathrm{DC} \cup \overline{\mathrm{NTPP}}), \mathcal{B})$,

$-\mathfrak{C}(N)^{w z}=(\mathrm{PO} \cup \mathrm{TPP} \cup \mathrm{NTPP}, \mathrm{TPP} \cup \mathrm{NTPP} \cup \mathrm{EQ}, \mathrm{NTPP}, \mathrm{TPP} \cup \mathrm{NTPP} \cup$ $\mathrm{EQ})$,

- $\mathfrak{C}(N)^{u x}=(\mathcal{B} \backslash \mathrm{DC}, \mathcal{B} \backslash(\mathrm{DC} \cup \mathrm{EC}), \mathrm{TPP} \cup \mathrm{NTPP} \cup \mathrm{EQ}, \mathrm{NTPP})$,

$-\mathfrak{C}(N)^{u y}=(\mathcal{B} \backslash(\mathrm{DC} \cup \mathrm{EC}), \mathcal{B} \backslash(\mathrm{DC} \cup \mathrm{EC}), \mathrm{PO} \cup \overline{\mathrm{TPP}} \cup \mathrm{EQ}, \overline{\mathrm{TPP}})$,

- $\mathfrak{C}(N)^{u z}=(\mathcal{B} \backslash(\mathrm{DC} \cup \mathrm{EC}), \mathcal{B} \backslash(\mathrm{DC} \cup \mathrm{EC}), \mathcal{B} \backslash \mathrm{DC}, \mathcal{B} \backslash \mathrm{DC})$,

- $\mathfrak{C}(N)^{u w}=(\mathcal{B} \backslash \mathrm{DC}, \mathcal{B}, \mathcal{B}, \mathcal{B}), \mathfrak{C}(N)^{v x}=(\mathcal{B} \backslash \mathrm{DC}, \mathcal{B} \backslash(\mathrm{DC} \cup \mathrm{EC} \cup \mathrm{NTPP}), \mathrm{PO} \cup$ $\mathrm{TPP} \cup \mathrm{EQ}, \mathrm{TPP})$,

$-\mathfrak{C}(N)^{v y}=(\mathcal{B} \backslash(\mathrm{DC} \cup \mathrm{EC}), \mathcal{B} \backslash(\mathrm{DC} \cup \mathrm{EC} \cup \mathrm{NTPP}), \mathrm{PO} \cup \overline{\mathrm{TPP}}, \mathrm{EC} \cup \mathrm{PO} \cup$ $\overline{\mathrm{TPP}} \cup \overline{\mathrm{NTPP}})$,

- $\mathfrak{C}(N)^{v z}=(\mathcal{B} \backslash(\mathrm{DC} \cup \mathrm{EC}), \mathcal{B} \backslash(\mathrm{DC} \cup \mathrm{EC}), \mathcal{B} \backslash \mathrm{DC}, \mathcal{B})$,

- $\mathfrak{C}(N)^{v w}=(\mathcal{B} \backslash \mathrm{DC}, \mathcal{B}, \mathcal{B}, \mathcal{B})$

$-\mathfrak{C}(N)^{v u}=(\overline{\mathrm{TPP}} \cup \overline{\mathrm{NTPP}} \cup \mathrm{EQ}, \overline{\mathrm{NTPP}}, \overline{\mathrm{TPP}} \cup \overline{\mathrm{NTPP}} \cup \mathrm{EQ}, \mathrm{PO} \cup \overline{\mathrm{TPP}} \cup$ $\overline{\mathrm{NTPP}})$.

However, $N$ is not satisfiable since $\mathfrak{C}(N)$ is not satisfiable. Indeed, to refine $\mathfrak{C}(N)_{2}^{x y}$ by EQ, TPP, or NTPP and then to apply the algebraic closure gives a trivially unsatisfiable network. This can be seen by the fact that the only satisfiable basic relations $B$ of $\mathfrak{C}(N)^{x y}$ satisfy $B_{2}=\mathrm{EQ}$ or $B_{3}=\mathrm{EQ}$ and that there exists neither algebraically closed scenario $S \subseteq \mathfrak{C}(N)_{2}$ satisfying $S^{x y}=$ EQ nor algebraically closed scenario $S \subseteq \mathfrak{C}(N)_{3}$ satisfying $S^{x y}=$ EQ. For example, by setting $\mathfrak{C}(N)_{2}^{x y}=$ EQ, we get $\mathfrak{C}(N)_{2}^{x y}=\mathfrak{C}(N)_{2}^{y z}=\mathfrak{C}(N)_{2}^{x z}=$ EQ and therefore $\mathfrak{C}(N)_{2}^{w x}=\mathfrak{C}(N)_{2}^{w y}=$ $\mathfrak{C}(N)_{2}^{w z}=\mathfrak{C}(N)_{2}^{w x} \cap \mathfrak{C}(N)_{2}^{w y} \cap \mathfrak{C}(N)_{2}^{w z}$ i.e. $(\mathrm{PO} \cup \overline{\mathrm{TPP}} \cup \mathrm{EQ}) \cap(\mathrm{PO} \cup \mathrm{TPP}) \cap$ $(\mathrm{TPP} \cup \mathrm{NTPP} \cup \mathrm{EQ})=\varnothing$.

Let $\mathcal{S}$ be a $\risingdotseq$-closed Cartesian subalgebra of $\mathrm{TRCC}_{8}^{\mathrm{n}}$ with $m=2$. The network $N^{\prime}$ satisfying $\mathrm{E}=\{u, v, w, x, y, z\}, N_{1}^{\prime}=\mathfrak{C}(N)_{2}$, and $N_{2}^{\prime}=\mathfrak{C}(N)_{3}$ is algebraically consistent, unsatisfiable, and over $\mathcal{S}$. 
For this reason, we are looking for Cartesian subclasses that do not contain all basic relations. In particular, we are interested in the following subset of $\mathrm{RCA}_{8}: \mathcal{H}_{8}^{\mathrm{NTPP}} \Rightarrow \mathrm{TPP}$ defined by $\left\{r \in \mathcal{H}_{8} \mid \mathrm{NTPP} \subseteq r \Rightarrow \mathrm{TPP} \subseteq r \wedge \overline{\mathrm{NTPP}} \subseteq r \Rightarrow \overline{\mathrm{TPP}} \subseteq r\right\}$. It is easy to prove that this subset is a subclass.

Lemma 1. The subset $\mathcal{H}_{8}^{\mathrm{NTPP} \Rightarrow \mathrm{TPP}}$ is a subclass.

We show that we can obtain algebraically tractable Cartesian subclasses of TRCC $_{8}^{\text {n }}$ satisfying $\mathcal{S}_{i} \in\left\{\mathcal{Q}_{8}, \mathcal{H}_{8}^{\mathrm{NTPP} \Rightarrow \mathrm{TPP}}\right\}$. For this, we apply the refinement theorem (by using the refinement $H_{\mathcal{S}}$; see Section 2.5. We begin by showing the conditions of the theorem.

Lemma 2. We have the following properties:

- $\forall r \in \mathcal{Q}_{8}, \uparrow r \in \mathcal{H}_{8}^{\mathrm{NTPP} \Rightarrow \mathrm{TPP}}$ and

- $\forall r \in \mathcal{H}_{8}^{\mathrm{NTPP}} \Rightarrow \mathrm{TPP}, \uparrow r \in \mathcal{Q}_{8}$.

Proof. From the definitions of $\mathcal{H}_{8}, \mathcal{Q}_{8}$, and the projections of $\mathrm{TRCC}_{8}^{\mathrm{n}}$, we derive the lemma. We show on the one hand that for any $r \in \mathcal{Q}_{8}$, we have $\uparrow r \in \mathcal{H}_{8}^{\mathrm{NTPP} \Rightarrow \mathrm{TPP}}$. Let $r \in \mathcal{Q}_{8}$. If PO $\nsubseteq \uparrow r$ then $r \subseteq \mathrm{DC} \cup \mathrm{NTPP} \cup \overline{\mathrm{NTPP}}$ (only DC, NTPP, and NTPP satisfy PO $\nsubseteq \uparrow b$ with $b \in \mathcal{B}_{\mathrm{RCC}_{8}}$ ). Therefore, $r$ is DC, NTPP, NTPP, DC $\cup$ NTPP, or DC $\cup \overline{\mathrm{NTPP}}$ (since $r \in \mathcal{Q}_{8}$ ). Thus, $\uparrow r \in \mathcal{H}_{8}^{\mathrm{NTPP} \Rightarrow \text { TPP }}$. Suppose PO $\subseteq \uparrow r$. If $\mathrm{NTPP} \nsubseteq \uparrow r$ and $\overline{\mathrm{NTPP}} \nsubseteq \uparrow r$ then $\uparrow r \in \mathcal{H}_{8}^{\mathrm{NTPP} \Rightarrow \mathrm{TPP}}$. Otherwise, if NTPP $\subseteq \uparrow r$ then either $\mathrm{TPP} \subseteq r$ or $\mathrm{NTPP} \subseteq r$ or $\mathrm{EQ} \subseteq r$. In all cases, TPP $\subseteq \uparrow r$. Similarly, if $\overline{\mathrm{NTPP}} \subseteq \uparrow r$ then $\overline{\mathrm{TPP}} \subseteq \uparrow r$. Therefore, in all cases, we have $\uparrow r \in \mathcal{H}_{8}^{\mathrm{NTPP}} \Rightarrow \mathrm{TPP}$.

We show on the other hand that for any $r \in \mathcal{H}_{8}^{\mathrm{NTPP}} \Rightarrow \mathrm{TPP}$, we have $\uparrow r \in \mathcal{Q}_{8}$. Let $r \in \mathcal{H}_{8}^{\mathrm{NTPP} \Rightarrow \mathrm{TPP}}$. If PO $\nsubseteq \uparrow r$ then $r \subseteq \mathrm{DC} \cup \mathrm{NTPP} \cup \overline{\mathrm{NTPP}}$ and therefore $r=\mathrm{DC}$. Thus, $\uparrow r=\mathrm{DC} \cup \mathrm{EC}$ and therefore $\uparrow r \in \mathcal{Q}_{8}$. If $\mathrm{PO} \subseteq \uparrow r$, we have $\uparrow r \in \mathcal{Q}_{8}$ by definition.

Lemma 3. Let $\mathcal{S} \in\left\{\mathcal{Q}_{8} \times \mathcal{Q}_{8}, \mathcal{Q}_{8} \times \mathcal{H}_{8}, \mathcal{H}_{8} \times \mathcal{Q}_{8}\right\}$ be a subclass of $\mathrm{TRCC}_{8}^{\mathrm{n}}(m=$ 2) and let $R \in \mathcal{S}$.

If $R$ is $ґ$-consistent then $H_{\mathcal{S}}(R)$ is $ґ$-consistent.

Proof. From the definitions of $\mathcal{H}_{8}, \mathcal{Q}_{8}, h_{\mathcal{H}_{8}}$, and the projections of $\mathrm{TRCC}_{8}^{\mathrm{n}}$, we derive the lemma. Let $\mathcal{S} \in\left\{\mathcal{Q}_{8} \times \mathcal{Q}_{8}, \mathcal{Q}_{8} \times \mathcal{H}_{8}, \mathcal{H}_{8} \times \mathcal{Q}_{8}\right\}$ and $R \in \mathcal{S}$ a $ґ$-consistent relation. We show that $H_{\mathcal{S}}(R)$ is $户$-consistent.

- If $\mathrm{DC} \subseteq R_{1}$ then $R_{2} \cap(\mathrm{DC} \cup \mathrm{EC}) \neq \varnothing$ and therefore $H_{\mathcal{S}}(R)=(\mathrm{DC}, \mathrm{DC})$ or $H_{\mathcal{S}}(R)=(\mathrm{DC}, \mathrm{EC})$.

- Otherwise, if EC $\subseteq R_{1}$ then $R_{2} \cap(\mathrm{DC} \cup \mathrm{EC} \cup \mathrm{PO}) \neq \varnothing$ and therefore $H_{\mathcal{S}}(R) \in$ $\{(\mathrm{EC}, \mathrm{DC}),(\mathrm{EC}, \mathrm{EC}),(\mathrm{EC}, \mathrm{PO})\}$.

- Otherwise, if PO $\subseteq R_{1}$ then $R_{2} \cap(\mathrm{EC} \cup \mathrm{PO} \cup \mathrm{TPP} \cup \mathrm{EQ} \cup \overline{\mathrm{TPP}}) \neq \varnothing$ and $\mathrm{DC} \nsubseteq R_{2}$.

- If $R_{2} \cap(\mathrm{EC} \cup \mathrm{PO} \cup \mathrm{TPP} \cup \overline{\mathrm{TPP}}) \neq \varnothing$, then $H_{\mathcal{S}}(R)_{1}=\mathrm{PO}$ and $H_{\mathcal{S}}(R)_{2} \in$ $\{\mathrm{EC}, \mathrm{PO}, \mathrm{TPP}, \overline{\mathrm{TPP}}\}$.

- Otherwise, EQ $\subseteq R_{2}$ and $R_{2} \subseteq$ EQ $\cup$ NTPP $\cup \overline{\mathrm{NTPP}}$ and therefore $R_{2}=\mathrm{EQ}$ $\left(R_{2} \in \mathcal{H}_{8} \cup \mathcal{Q}_{8}\right)$. Thus, $H_{\mathcal{S}}(R)=(\mathrm{PO}, \mathrm{EQ})$. 
- Otherwise, if TPP $\subseteq R_{1}$ then $R_{2} \cap(\mathrm{PO} \cup \mathrm{TPP} \cup \mathrm{NTPP} \cup \mathrm{EQ}) \neq \varnothing, R_{2} \cap$ $(\mathrm{DC} \cup \mathrm{EC})=\varnothing$ and $R_{1} \subseteq \mathrm{TPP} \cup \mathrm{NTPP} \cup \mathrm{EQ}\left(R_{1} \in \mathcal{H}_{8} \cup \mathcal{Q}_{8}\right)$.

- If $R_{2} \cap(\mathrm{PO} \cup \mathrm{TPP}) \neq \varnothing$ then $H_{\mathcal{S}}(R)$ is either (TPP, PO) or (TPP, TPP).

- Otherwise, $R_{2} \subseteq \mathrm{NTPP} \cup \mathrm{EQ} \cup \overline{\mathrm{TPP}} \cup \overline{\mathrm{NTPP}}$.

* If NTPP $\subseteq R_{2}$ then $R_{2}=\operatorname{NTPP}\left(R_{2} \in \mathcal{H}_{8} \cup \mathcal{Q}_{8}\right)$ and therefore $H_{\mathcal{S}}(R)=(\mathrm{TPP}, \mathrm{NTPP})$.

* If $\mathrm{EQ} \subseteq R_{2}$ then $R_{2} \subseteq \overline{\mathrm{TPP}} \cup \overline{\mathrm{NTPP}} \cup \mathrm{EQ}\left(R_{2} \in \mathcal{H}_{8} \cup \mathcal{Q}_{8}\right)$.

- If $\mathcal{S}_{1}=\mathcal{Q}_{8}$ then $R_{1} \subseteq \mathrm{TPP} \cup \mathrm{NTPP}$ and therefore $R_{2}=\mathrm{EQ}$. Thus, $H_{\mathcal{S}}(R)=(\mathrm{TPP}, \mathrm{EQ})$.

. If $\mathcal{S}_{2}=\mathcal{Q}_{8}$ then $R_{2}=\mathrm{EQ}$ and therefore $H_{S}(R)=$ (TPP. EQ).

- Otherwise, if $\overline{\mathrm{TPP}} \subseteq R_{1}$ then $R_{2} \cap(\mathrm{DC} \cup \mathrm{EC})=\varnothing_{2}, R_{2} \cap(\mathrm{PO} \cup \overline{\mathrm{TPP}} \cup \overline{\mathrm{NTPP}} \cup$ $\mathrm{EQ}) \neq \varnothing$ and $R_{1} \subseteq \overline{\mathrm{TPP}} \cup \overline{\mathrm{NTPP}} \cup \mathrm{EQ}\left(R_{1} \in \mathcal{H}_{8} \cup \mathcal{Q}_{8}\right)$.

- If $\mathrm{PO} \subseteq R_{2}$ then $H_{\mathcal{S}}(R)=(\overline{\mathrm{TPP}}, \mathrm{PO})$.

- Otherwise, either $R_{2} \subseteq \overline{\mathrm{TPP}} \cup \overline{\mathrm{NTPP}} \cup \mathrm{EQ}$ or $R_{2} \subseteq \mathrm{TPP} \cup \mathrm{NTPP} \cup \mathrm{EQ}$ and $\mathrm{EQ} \subseteq R_{2}\left(R_{2} \in \mathcal{H}_{8} \cup \mathcal{Q}_{8}\right)$.

* If $\mathcal{S}_{1}=\mathcal{Q}_{8}$ then $R_{1} \subseteq \overline{\mathrm{TPP}} \cup \overline{\mathrm{NTPP}}$ and therefore either $R_{2} \subseteq \overline{\mathrm{TPP}} \cup$ $\overline{\mathrm{NTPP}} \cup \mathrm{EQ}$ or $R_{2}=\mathrm{EQ}$. Thus, $H_{\mathcal{S}}(R)$ is $(\overline{\mathrm{TPP}}, \overline{\mathrm{TPP}}),(\overline{\mathrm{TPP}}, \overline{\mathrm{NTPP}})$, or ( $\overline{\mathrm{TPP}}, \mathrm{EQ})$.

* If $\mathcal{S}_{2}=\mathcal{Q}_{8}$ then either $R_{2} \subseteq \overline{\mathrm{TPP}} \cup \overline{\mathrm{NTPP}}$ or $R_{2}=\mathrm{EQ}$. Thus, $H_{\mathcal{S}}(R)=$ $(\overline{\mathrm{TPP}}, \overline{\mathrm{TPP}})$ or $H_{\mathcal{S}}(R)=(\overline{\mathrm{TPP}}, \overline{\mathrm{NTPP}})$ or $H_{\mathcal{S}}(R)=(\overline{\mathrm{TPP}}, \mathrm{EQ})$.

- Otherwise $R_{1} \subseteq \mathrm{NTPP} \cup \mathrm{EQ} \cup \overline{\mathrm{NTPP}}$ :

- If NTPP $\subseteq R_{1}$ then $R_{1}=\operatorname{NTPP}\left(R_{1} \in \mathcal{H}_{8} \cup \mathcal{Q}_{8}\right)$ and $R_{2} \subseteq \mathrm{TPP} \cup$ $\mathrm{NTPP} \cup \mathrm{EQ}$. Thus, $H_{\mathcal{S}}(R)=(\mathrm{NTPP}, \mathrm{TPP})$ or $H_{\mathcal{S}}(R)=(\mathrm{NTPP}, \mathrm{NTPP})$ or $H_{\mathcal{S}}(R)=(\mathrm{NTPP}, \mathrm{EQ})$.

- If $\overline{\mathrm{NTPP}} \subseteq R_{1}$ then $R_{1}=\overline{\mathrm{NTPP}}\left(R_{1} \in \mathcal{H}_{8} \cup \mathcal{Q}_{8}\right)$ and $R_{2} \subseteq \overline{\mathrm{TPP}} \cup$ $\overline{\mathrm{NTPP}} \cup \mathrm{EQ}$. Thus, $H_{\mathcal{S}}(R)=(\overline{\mathrm{NTPP}}, \overline{\mathrm{TPP}})$ or $H_{\mathcal{S}}(R)=(\overline{\mathrm{NTPP}}, \overline{\mathrm{NTPP}})$ or $H_{\mathcal{S}}(R)=(\overline{\mathrm{NTPP}}, \mathrm{EQ})$.

- If $\mathrm{EQ} \subseteq R_{1}$ then $R_{1}=\mathrm{EQ}\left(R_{1} \in \mathcal{H}_{8} \cup \mathcal{Q}_{8}\right)$ and $R_{2} \subseteq \mathrm{PO} \cup \mathrm{TPP} \cup \mathrm{NTPP} \cup$ $\mathrm{EQ} \cup \overline{\mathrm{TPP}} \cup \overline{\mathrm{NTPP}}$. Thus, $H_{\mathcal{S}}(R)_{1}=\mathrm{EQ}$ and $H_{\mathcal{S}}(R)_{2}$ is either PO, TPP, NTPP, $\overline{\mathrm{TPP}}, \overline{\mathrm{NTPP}}$, or EQ.

Note the following proposition, before identifying the tractable subclasses. It shows that although the tractable subalgebras of $\mathrm{RCC}_{8}$ cannot be combined to obtain algebraically tractable Cartesian subalgebras, the algebraically consistent networks over the majority of these combinations are satisfiable.

Proposition 2. Let $\mathfrak{R}$ be a topological region space such that $\mathrm{TRCC}_{8}^{\mathrm{n}}$ is a complete sequential formalism. Let $\mathcal{S}$ be a subset of $\mathrm{TRCC}_{8}^{\mathrm{n}}$ satisfying $\mathcal{S}_{i} \in\left\{\mathcal{H}_{8}, \mathcal{Q}_{8}\right\}$ and $\mathcal{S}_{i}=\mathcal{H}_{8} \Longrightarrow\left(i=m \vee \mathcal{S}_{i+1}=\mathcal{Q}_{8}\right) \wedge\left(i=1 \vee \mathcal{S}_{i-1}=\mathcal{Q}_{8}\right)$ for all $i \in \mathrm{I}$.

Algebraically consistent networks over $\mathcal{S}$ are satisfiable.

Proof. We apply the refinement theorem [7]. $H_{\mathcal{S}}$ is a refinement from $\mathcal{S}$ to the set $\mathcal{B} \cup\{(\varnothing, \ldots, \varnothing)\}$ (since $h_{\mathcal{H}_{8}}$ is a refinement from $\mathcal{H}_{8} \cup \mathcal{Q}_{8}$ to $\mathcal{B}_{\mathrm{RCC}_{8}} \cup\{\varnothing\}$ [38]). $\mathcal{S}$ is algebraically stable by $H_{\mathcal{S}}$. Indeed, since on the one hand, $\mathcal{H}_{8}$ and $\mathcal{Q}_{8}$ are algebraically stable by $h_{\mathcal{H}_{8}}$ [38]. Since, on the other hand, for any $\risingdotseq$-consistent relation $R \in \mathcal{S}, H_{\mathcal{S}}(R)$ is $\risingdotseq$-consistent (by Lemma 3). Algebraically consistent networks over $\mathcal{B} \cup\{(\varnothing, \ldots, \varnothing)\}$ are satisfiable ( $\operatorname{TRCC}_{8}^{\mathrm{n}}$ is complete). By the refinement theorem, algebraically consistent networks over $\mathcal{S}$ are satisfiable. 
Satisfiability of algebraically consistent networks is, in general, a weaker property than algebraic tractability. It is not equivalent for subclasses that are not $\risingdotseq$-closed. Indeed, applying the algebraic closure on a network over a subclass which is not $\risingdotseq$-closed can move the network out of the subclass. In that case, we cannot therefore conclude that the network is satisfiable (if it is not trivially inconsistent). The previous subclasses are not $\risingdotseq$-closed: the projection of NTPP, $\uparrow \mathrm{NTPP}=\mathrm{TPP} \cup \mathrm{NTPP} \cup \mathrm{EQ}$ does not belong to $\mathcal{Q}_{8}$.

We end this section by showing that the subclasses of the following particular forms $\left(\mathcal{Q}_{8} \times \mathcal{H}_{8}^{\mathrm{NTPP}} \Rightarrow \mathrm{TPP}\right){ }^{\star},\left(\mathcal{H}_{8}^{\mathrm{NTPP} \Rightarrow \mathrm{TPP}} \times \mathcal{Q}_{8}\right)^{\star}, \mathcal{H}_{8}^{\mathrm{NTPP} \Rightarrow \mathrm{TPP}} \times\left(\mathcal{Q}_{8} \times \mathcal{H}_{8}^{\mathrm{NTPP} \Rightarrow \mathrm{TPP}}\right)^{\star}$, and $\mathcal{Q}_{8} \times\left(\mathcal{H}_{8}^{\mathrm{NTPP}} \Rightarrow \mathrm{TPP} \times \mathcal{Q}_{8}\right)^{\star}$ are algebraically tractable.

Proposition 3. Let $\mathfrak{R}$ be a topological region space such that $\mathrm{TRCC}_{8}^{\mathrm{n}}$ is a complete sequential formalism. Let $\mathcal{S}$ be a subset of $\mathrm{TRCC}_{8}^{\mathrm{n}}$ satisfying one of the two following properties:

$-\mathcal{S}_{i}=\mathcal{H}_{8}^{\mathrm{NTPP} \Rightarrow \mathrm{TPP}}$ if $i$ is even and $\mathcal{S}_{i}=\mathcal{Q}_{8}$ otherwise, for all $i \in \mathrm{I}$,

- $\mathcal{S}_{i}=\mathcal{H}_{8}^{\mathrm{NPP}} \Rightarrow \mathrm{TPP}$ if $i$ is odd and $\mathcal{S}_{i}=\mathcal{Q}_{8}$ otherwise, for all $i \in \mathrm{I}$.

The subclass $\mathcal{S}$ is algebraically tractable.

Proof. Let $\mathcal{S}$ be a subset of $\mathrm{TRCC}_{8}^{\mathrm{n}}$ as described in the statement. $\mathcal{S}$ satisfies the conditions of the first part of the refinement theorem (see the proof of Proposition 2). $\mathcal{S}$ also satisfies the conditions of the second part. Indeed, on the one hand, $\mathcal{S}$ is a subclass (since $\mathcal{S}$ is Cartesian and each $\mathcal{S}_{i}$ is a subclass [38] ; Lemma 11. On the other hand, $\mathcal{S}$ is $户$-closed (by Lemma 2 and since $\mathcal{S}$ is a Cartesian subclass). $\mathcal{S}$ is thus algebraically tractable (refinement theorem [7]).

Note that the tractable subclasses identified by Proposition 3 do not contain all the basic relations (thus, they are not subalgebras).

\section{Topological Sequences on a Partition of Time}

We have shown that there is no algebraically tractable Cartesian subalgebra in the context of $\mathrm{TRCC}_{8}^{\mathrm{n}}$, the context of regions described at different time points between which there are no intermediary relations (i.e. at time points which characterize all the qualitative changes). Does this mean that there are no large tractable subclasses in the context of (topological) temporal sequences? We show that this is not the case, by considering topological temporal sequences describing the evolution of regions on a time partition (i.e. on a contiguous alternation of instants and open intervals).

\subsection{Formalization}

We begin by defining the formalism of topological temporal sequences on a partition of time, which we denote $\mathrm{TRCC}_{8}^{\mathrm{d}}$. Without loss of generality, we consider only the partitions of the interval $\left[t_{0}, t_{l}\left[\right.\right.$ of the form $\left(t_{0},\right] t_{0}, t_{1}\left[, \ldots, t_{l-1},\right] t_{l-1}, t_{l}[)$ with $m=2 l$, $t_{i-1}<t_{i}$ for all $i \in\{1, \ldots, l\}, t_{i} \in \mathbb{R}$ for all $i \in\{0, \ldots, l\}$, and $l \in \mathbb{N}^{*}$. Thus, the sequences of $\mathrm{TRCC}_{8}^{\mathrm{d}}$ describe the topological relations at each time periods $t_{i}$ and during each interval $] t_{i}, t_{i+1}[$. 
Definition 4. Let $(\Re, T)$ be a topological region space. Let $t_{0}, \ldots, t_{l} \in \mathbb{R}$ be consecutive instants.

The formalism $\operatorname{TRCC}_{8}^{\mathrm{d}}$ (associated with $(\mathfrak{R}, T)$ ) is the triplet $(\mathcal{A}, \mathcal{U}, \varphi)$ where:

- $\mathcal{U}$ is the set of continuous functions from $\left[t_{1}, t_{m}[\right.$ to the set of regions $\mathfrak{R}$,

- $\mathcal{A}$ is the multi-algebra whose Cartesian product is $\mathrm{RCA}_{8}^{m}$ and whose projections satisfy $\Gamma_{i}^{j} b=\uparrow b$ if $|j-i|=1$ and $i$ is even, $\Gamma_{i}^{j} b=\downarrow b$ if $|j-i|=1$ and $i$ is odd, and $\Gamma_{i}^{j} b=\mathcal{B}_{\mathrm{RCC}_{8}}$ otherwise, for all $b \in \mathcal{B}_{\mathrm{RCC}_{8}}$ and $i, j \in \mathrm{I}$ with $\uparrow$ and $\downarrow$ defined by Table 4 and

- $\varphi$ is the function from $\mathcal{A}$ to $2^{\mathcal{U} \times \mathcal{U}}$ such that for all $R \in \mathcal{A}, \varphi(R)$ is the set of pairs of functions $\left(f, f^{\prime}\right) \in \mathcal{U} \times \mathcal{U}$ satisfying at each instant $t_{i}$ the relation $R_{2 i+1}$ (i.e. $\left.\forall i \in\{0, \ldots, l-1\}\left(f\left(t_{i}\right), f^{\prime}\left(t_{i}\right)\right) \in \varphi_{\mathrm{RCC}_{8}}\left(R_{2 i+1}\right)\right)$ and satisfying, for each $i \in\{0, \ldots, l-1\}$, one (and only one) basic relation $b \subseteq R_{2 i+2}$ at each instant between $t_{i}$ and $t_{i+1}$ (i.e. $\left.\exists b \in \mathcal{B}_{\mathrm{RCC}_{8}} b \subseteq R_{2 i+2} \forall t \in\right] t_{i}, t_{i+1}\left[\left(f(t), f^{\prime}(t)\right) \in\right.$ $\varphi_{\mathrm{RCC}_{8}}(b)$ ), with $\varphi_{\mathrm{RCC}_{8}}$ the interpretation function of $\mathrm{RCC}_{8}$.

Remark 3. The operator $\uparrow$ encodes the dominance graph of $\mathrm{RCC}_{8}$ described in Figure $2 \mathrm{~b}$, i.e. the possible evolutions of relations being satisfied during an open interval ( $\uparrow$ returns the corresponding relations possibly satisfied at the limits of the interval). The operators $\uparrow$ and $\downarrow$ enforces continuity (when $\mathrm{TRCC}_{8}^{\mathrm{d}}$ is a complete sequential formalism). Remark 1 on $\mathrm{TRCC}_{8}^{\mathrm{n}}$ also applies to $\mathrm{TRCC}_{8}^{\mathrm{d}}$.

Example 2. An example of relations of $\mathrm{TRCC}_{8}^{\mathrm{d}}$, with $m=4$, is the relation $R=$ $(\mathrm{TPP} \cup \mathrm{NTPP}, \mathrm{PO} \cup \mathrm{EQ}, \mathrm{EC} \cup \mathrm{DC}, \mathrm{DC})$. This relation means that the first region is included in the second at the instant $t_{0}$ ( $R_{1}$ is satisfied), then they overlap during the interval $] t_{0}, t_{1}[$ or are equal during the interval $] t_{0}, t_{1}\left[\left(R_{2}\right.\right.$ is satisfied), they are disjoined at the instant $t_{1}\left(R_{3}\right.$ is satisfied), and finally they are disconnected at every instant of $] t_{1}, t_{2}\left[\right.$ ( $R_{4}$ is satisfied). The only satisfiable basic relation included in $R$ is $(\mathrm{TPP}, \mathrm{PO}, \mathrm{EC}, \mathrm{DC})=\longmapsto(R)$.

\begin{tabular}{|c|c|c|}
\hline$b$ & $\uparrow b$ & $\downarrow b$ \\
\hline \hline $\mathrm{DC}$ & $\mathrm{DC} \cup \mathrm{EC}$ & $\mathrm{DC}$ \\
\hline $\mathrm{EC}$ & $\mathrm{EC}$ & $\mathrm{DC} \cup \mathrm{EC} \cup \mathrm{PO}$ \\
\hline $\mathrm{PO}$ & $\mathrm{EC} \cup \mathrm{PO} \cup \mathrm{TPP} \cup \overline{\mathrm{TPP}} \cup \mathrm{EQ}$ & $\mathrm{PO}$ \\
\hline $\mathrm{TPP}$ & $\mathrm{TPP} \cup \mathrm{EQ}$ & $\mathrm{PO} \cup \mathrm{TPP} \cup \mathrm{NTPP}$ \\
\hline $\mathrm{NTPP}$ & $\mathrm{NTPP} \cup \mathrm{TPP} \cup \mathrm{EQ}$ & $\mathrm{NTPP}$ \\
\hline $\mathrm{EQ}$ & $\mathrm{EQ}$ & $\mathcal{B}_{\mathrm{RCC}_{8}} \backslash(\mathrm{DC} \cup \mathrm{EC})$ \\
\hline
\end{tabular}

Table 4. Dominant and dominated relations of the basic relations of $\mathrm{RCC}_{8}$.

\subsection{Tractability Results}

We now identify large algebraically tractable Cartesian subalgebras, by applying again the refinement theorem. We begin by showing its conditions. 
Lemma 4. Let $\mathcal{S} \in\left\{\mathcal{H}_{8} \times \mathcal{H}_{8}, \mathcal{H}_{8} \times \mathcal{Q}_{8}, \mathcal{Q}_{8} \times \mathcal{H}_{8}, \mathcal{Q}_{8} \times \mathcal{Q}_{8}, \mathcal{H}_{8} \times \mathcal{C}_{8}, \mathcal{Q}_{8} \times \mathcal{C}_{8}\right\}$ be a subclass of $\operatorname{TRCC}_{8}^{\mathrm{d}}(m=2)$ and $R \in \mathcal{S}$.

If $R$ is ¡-consistent then $H_{\mathcal{S}}(R)$ is 户-consistent.

Proof. From the definitions of $\mathcal{H}_{8}, \mathcal{Q}_{8}, \mathcal{C}_{8}, h_{\mathcal{H}_{8}}, h_{\mathcal{C}_{8}}$, and the projections of $\mathrm{TRCC}_{8}^{\mathrm{d}}$, we derive the lemma. Let $\mathcal{S} \in\left\{\mathcal{H}_{8} \times \mathcal{H}_{8}, \mathcal{H}_{8} \times \mathcal{Q}_{8}, \mathcal{Q}_{8} \times \mathcal{H}_{8}, \mathcal{Q}_{8} \times \mathcal{Q}_{8}, \mathcal{H}_{8} \times \mathcal{C}_{8}, \mathcal{Q}_{8} \times \mathcal{C}_{8}\right\}$ and $R \in \mathcal{S}$ be a $ґ$-consistent relation. We show that $H_{\mathcal{S}}(R)$ is $ґ$-consistent.

- If $\mathrm{DC} \subseteq R_{1}$ then $\mathrm{DC} \subseteq R_{2}$ and therefore $H_{\mathcal{S}}(R)=(\mathrm{DC}, \mathrm{DC})$.

- Otherwise, if $\mathrm{EC} \subseteq R_{1}$ then $R_{2} \cap(\mathrm{DC} \cup \mathrm{EC} \cup \mathrm{PO}) \neq \varnothing$.

- If $\mathcal{S}_{2} \in\left\{\mathcal{H}_{8}, \mathcal{Q}_{8}\right\}$ then $H_{\mathcal{S}}(R)_{1}=\mathrm{EC}$ and $H_{\mathcal{S}}(R)_{2} \subseteq \mathrm{DC} \cup \mathrm{EC} \cup \mathrm{PO}$.

- Suppose $\mathcal{S}_{2}=\mathcal{C}_{8}$.

* If $\mathrm{DC} \nsubseteq R_{2}$ and $\mathrm{PO} \nsubseteq R_{2}$ then $\mathrm{EC} \subseteq R_{2}$ and therefore $R_{2}=\mathrm{EC}$ (since $\left.R_{2} \in \mathcal{C}_{8}\right)$. Thus, $H_{\mathcal{S}}(R)=(\mathrm{EC}, \mathrm{EC})$

* If DC $\subseteq R_{2}$ or $\mathrm{PO} \subseteq R_{2}$ then $H_{\mathcal{S}}(R)_{1}=\mathrm{EC}$ and $H_{\mathcal{S}}(R)_{2} \subseteq \mathrm{DC} \cup \mathrm{PO}$.

- Otherwise, if $\mathrm{PO} \subseteq R_{1}$ then $\mathrm{PO} \subseteq R_{2}$ and $R_{2} \cap(\mathrm{DC} \cup \mathrm{EC})=\varnothing$ (since $R_{1} \cap$ $(\mathrm{DC} \cup \mathrm{EC})=\varnothing)$. Therefore, $H_{\mathcal{S}}(R)=(\mathrm{PO}, \mathrm{PO})$.

- Otherwise, if TPP $\subseteq R_{1}$ then $R_{2} \cap(\mathrm{TPP} \cup \mathrm{NTPP} \cup \mathrm{PO}) \neq \varnothing$ and $R_{2} \cap$ $(\mathrm{DC} \cup \mathrm{EC})=\varnothing$.

- Suppose $\mathcal{S}_{2} \in\left\{\mathcal{H}_{8}, \mathcal{Q}_{8}\right\}$.

* If $\mathrm{PO} \subseteq R_{2}$ or TPP $\subseteq R_{2}$ then $H_{\mathcal{S}}(R)=(\mathrm{TPP}, \mathrm{PO})$ or $H_{\mathcal{S}}(R)=$ (TPP, TPP).

* If $\mathrm{PO} \nsubseteq R_{2}$ and TPP $\nsubseteq R_{2}$ then NTPP $\subseteq R_{2}$ and therefore $R_{2}=$ NTPP (since $R_{2} \in \mathcal{H}_{8} \cup \mathcal{Q}_{8}$ ). Thus, $H_{\mathcal{S}}(R)=$ (TPP, NTPP).

- Suppose $\mathcal{S}_{2}=\mathcal{C}_{8}$.

* If $\mathrm{PO} \subseteq R_{2}$ or $\mathrm{NTPP} \subseteq R_{2}$ then $H_{\mathcal{S}}(R)=(\mathrm{TPP}, \mathrm{PO})$ or $H_{\mathcal{S}}(R)=$ (TPP, NTPP).

* If $\mathrm{PO} \nsubseteq R_{2}$ and NTPP $\nsubseteq R_{2}$ then TPP $\subseteq R_{2}$ and therefore $R_{2} \subseteq$ $\mathrm{TPP} \cup \mathrm{EQ}\left(R_{2} \in \mathcal{C}_{8}\right)$. Thus, $H_{\mathcal{S}}(R)=(\mathrm{TPP}, \mathrm{TPP})$.

- Otherwise, if $\overline{\mathrm{TPP}} \subseteq R_{1}$ then $R_{2} \cap(\overline{\mathrm{TPP}} \cup \overline{\mathrm{NTPP}} \cup \mathrm{PO}) \neq \varnothing$ and $R_{2} \cap$ $(\mathrm{DC} \cup \mathrm{EC})=\varnothing$.

- If $\mathrm{PO} \subseteq R_{2}$ then $H_{\mathcal{S}}(R)=(\overline{\mathrm{TPP}}, \mathrm{PO})$.

- If PO $\nsubseteq R_{2}$ then $R_{2} \subseteq \overline{\mathrm{TPP}} \cup \overline{\mathrm{NTPP}} \cup \mathrm{EQ}\left(R_{2} \in \mathcal{H}_{8} \cup \mathcal{Q}_{8} \cup \mathcal{C}_{8}\right)$.

* If $\mathcal{S}_{2} \in\left\{\mathcal{H}_{8}, \mathcal{Q}_{8}\right\}$ and $\overline{\mathrm{TPP}} \subseteq R_{2}$, we have $H_{\mathcal{S}}(R)=(\overline{\mathrm{TPP}}, \overline{\mathrm{TPP}})$.

* If $\mathcal{S}_{2} \in\left\{\mathcal{H}_{8}, \mathcal{Q}_{8}\right\}$ and $\overline{\mathrm{TPP}} \nsubseteq R_{2}$, we have EQ $\nsubseteq R_{2}$ (since $R_{2} \in$ $\left.\mathcal{H}_{8} \cup \mathcal{Q}_{8}\right)$. Therefore, $H_{\mathcal{S}}(R)=(\overline{\mathrm{TPP}}, \overline{\mathrm{NTPP}})$.

* If $\mathcal{S}_{2} \in \mathcal{C}_{8}$, since $R_{2} \cap(\overline{\mathrm{TPP}} \cup \overline{\mathrm{NTPP}}) \neq \varnothing$, we have $H_{\mathcal{S}}(R)=$ $(\overline{\mathrm{TPP}}, \overline{\mathrm{NTPP}})$ or $H_{\mathcal{S}}(R)=(\overline{\mathrm{TPP}}, \overline{\mathrm{TPP}})$.

- Otherwise, $R_{1} \subseteq \mathrm{NTPP} \cup \overline{\mathrm{NTPP}} \cup \mathrm{EQ}$ and $R_{2} \subseteq \mathrm{PO} \cup \mathrm{TPP} \cup \mathrm{NTPP} \cup \overline{\mathrm{TPP}} \cup$ $\overline{\mathrm{NTPP}} \cup \mathrm{EQ}$.

- If NTPP $\subseteq R_{1}$ then $R_{1}=\operatorname{NTPP}\left(R_{1} \in \mathcal{H}_{8} \cup \mathcal{Q}_{8}\right)$ and therefore $R_{2}=$ NTPP. Thus, $R=H_{\mathcal{S}}(R)=(\mathrm{NTPP}, \mathrm{NTPP})$.

- If $\overline{\mathrm{NTPP}} \subseteq R_{1}$ then, similarly, $R=H_{\mathcal{S}}(R)=(\overline{\mathrm{NTPP}}, \overline{\mathrm{NTPP}})$.

- Otherwise, $\mathrm{EQ}=R_{1}$. Therefore, $H_{\mathcal{S}}(R)_{1}=\mathrm{EQ}$ and $H_{\mathcal{S}}(R)_{2} \subseteq \mathrm{PO} \cup$ $\mathrm{TPP} \cup \mathrm{NTPP} \cup \overline{\mathrm{TPP}} \cup \overline{\mathrm{NTPP}} \cup \mathrm{EQ}$. 
Note that, as in the context of $\mathrm{TRCC}_{8}^{\mathrm{n}}$, algebraically consistent networks over most combinations of the subalgebras $\mathcal{Q}_{8}$ and $\mathcal{H}_{8}$, but also of $\mathcal{C}_{8}$, are satisfiable.

Proposition 4. Let $\mathfrak{R}$ be a topological region space such that $\mathrm{TRCC}_{8}^{\mathrm{d}}$ is a complete sequential formalism. Let $\mathcal{S}$ be a subset of $\mathrm{TRCC}_{8}^{\mathrm{d}}$ satisfying $S_{2 i-1} \in\left\{\mathcal{H}_{8}, \mathcal{Q}_{8}\right\}$ and $\mathcal{S}_{2 i} \in\left\{\mathcal{H}_{8}, \mathcal{Q}_{8}, \mathcal{C}_{8}\right\}$ for all $i \in\{1, \ldots, l\}$.

Algebraically consistent networks over $\mathcal{S}$ are satisfiable.

Proof. We apply the refinement theorem [7]. $H_{\mathcal{S}}$ is a refinement from $\mathcal{S}$ to the set $\mathcal{B} \cup\{(\varnothing, \ldots, \varnothing)\}$ (since $h_{\mathcal{H}_{8}}$ (resp. $h_{\mathcal{C}_{8}}$ ) is a refinement from $\mathcal{H}_{8} \cup \mathcal{Q}_{8}$ (resp. $h_{\mathcal{C}_{8}}$ ) to $\left.\mathcal{B}_{\mathrm{RCC}_{8}} \cup\{\varnothing\}[38]\right) . \mathcal{S}$ is algebraically stable by $H_{\mathcal{S}}$. Indeed, since on the one hand, $\mathcal{H}_{8}$ (resp. $\mathcal{Q}_{8} ;$ resp. $\mathcal{C}_{8}$ ) is algebraically stable by $h_{\mathcal{H}_{8}}$ (resp. $h_{\mathcal{H}_{8}} ;$ resp. $h_{\mathcal{C}_{8}}$ ) [38]. Since, on the other hand, for any $「$-consistent relation $R \in \mathcal{S}, H_{\mathcal{S}}(R)$ is $「$-consistent (by Lemma 4. Algebraically consistent networks over $\mathcal{B} \cup\{(\varnothing, \ldots, \varnothing)\}$ are satisfiable ( $\mathrm{TRCC}_{8}^{\mathrm{d}}$ is complete). By the refinement theorem, algebraically consistent networks over $\mathcal{S}$ are satisfiable.

Lemma 5. Let $r \in \mathrm{RCA}_{8} \backslash \mathcal{N}$. We have:

$$
\begin{aligned}
& \text { - } \uparrow r \in \mathcal{H}_{8}, \\
& \text { - } \downarrow r \in \mathcal{H}_{8} \cap \mathcal{Q}_{8} \cap \mathcal{C}_{8} .
\end{aligned}
$$

Proof. From the definitions of $\mathcal{H}_{8}, \mathcal{Q}_{8}, \mathcal{C}_{8}$ and the projections of $\mathrm{TRCC}_{8}^{\mathrm{d}}$, we derive the lemma. Let $r \in \mathrm{RCA}_{8} \backslash \mathcal{N}$ (see Table 2). On the one hand, we show $\uparrow r \in \mathcal{H}_{8}$. For this, we show the three following properties: $\uparrow r \in \mathrm{RCA}_{8} \backslash \mathcal{N}, \mathrm{NTPP} \subseteq \uparrow r \Longrightarrow \mathrm{TPP} \subseteq$ $\uparrow r$, and $\uparrow r \notin V=\{\mathrm{EC} \cup \mathrm{NTPP} \cup \mathrm{EQ}, \mathrm{DC} \cup \mathrm{EC} \cup \mathrm{NTPP} \cup \mathrm{EQ}, \mathrm{EC} \cup \overline{\mathrm{NTPP}} \cup$ $\mathrm{EQ}, \mathrm{DC} \cup \mathrm{EC} \cup \overline{\mathrm{NTPP}} \cup \mathrm{EQ}\}$. We show $\uparrow r \in \mathrm{RCA}_{8} \backslash \mathcal{N}$. If $\uparrow r \cap(\mathrm{TPP} \cup \mathrm{NTPP})=\varnothing$ or $\uparrow r \cap(\overline{\mathrm{TPP}} \cup \overline{\mathrm{NTPP}})=\varnothing$ then $\uparrow r \in \mathrm{RCA}_{8} \backslash \mathcal{N}$. Suppose $\uparrow r \cap(\mathrm{TPP} \cup \mathrm{NTPP}) \neq$ $\varnothing$ and $\uparrow r \cap(\overline{\mathrm{TPP}} \cup \overline{\mathrm{NTPP}}) \neq \varnothing$. Therefore, $r \cap(\mathrm{PO} \cup \mathrm{TPP} \cup \mathrm{NTPP}) \neq \varnothing$ and $r \cap$ $(\mathrm{PO} \cup \overline{\mathrm{TPP}} \cup \overline{\mathrm{NTPP}}) \neq \varnothing$. If $r \cap(\mathrm{TPP} \cup \mathrm{NTPP}) \neq \varnothing$ and $r \cap(\overline{\mathrm{TPP}} \cup \overline{\mathrm{NTPP}}) \neq$ $\varnothing$ then $\mathrm{PO} \subseteq r$ (since $r \in \mathrm{RCA}_{8} \backslash \mathcal{N}$ ). Thus, $\mathrm{PO} \subseteq r$ and therefore $\mathrm{PO} \subseteq \uparrow r$. We have $\uparrow r \in \mathrm{RCA}_{8} \backslash \mathcal{N}$. We show NTPP $\subseteq \uparrow r \Rightarrow \operatorname{TPP} \subseteq \uparrow r$. If NTPP $\subseteq \uparrow r$, then NTPP $\subseteq r$ and therefore $\uparrow \mathrm{NTPP} \subseteq \uparrow r$. Thus, TPP $\subseteq \uparrow r$. We show $\uparrow r \notin V$. If $\uparrow r \cap(\mathrm{NTPP} \cup \overline{\mathrm{NTPP}})=\varnothing$ then $\uparrow r \notin V$. If $\mathrm{NTPP} \subseteq \uparrow r$, then $\mathrm{TPP} \subseteq \uparrow r$. If $\overline{\mathrm{NTPP}} \subseteq \uparrow r$ then $\overline{\mathrm{TPP}} \subseteq \uparrow r$. Thus, in all cases, $\uparrow r \notin V$.

On the other hand, we show $\downarrow r \in \mathcal{H}_{8} \cap \mathcal{Q}_{8} \cap \mathcal{C}_{8}$. If $\downarrow r \cap(\mathrm{TPP} \cup \mathrm{NTPP})=\varnothing$ or $\downarrow r \cap(\overline{\mathrm{TPP}} \cup \overline{\mathrm{NTPP}})=\varnothing$ then $\downarrow r \in \mathrm{RCA}_{8} \backslash \mathcal{N}$. Suppose $\downarrow r \cap(\mathrm{TPP} \cup \mathrm{NTPP}) \neq$ $\varnothing$ and $\downarrow r \cap(\overline{\mathrm{TPP}} \cup \overline{\mathrm{NTPP}}) \neq \varnothing$. We have $r \cap(\mathrm{TPP} \cup \mathrm{NTPP} \cup \mathrm{EQ}) \neq \varnothing$ and $r \cap(\overline{\mathrm{TPP}} \cup \overline{\mathrm{NTPP}} \cup \mathrm{EQ}) \neq \varnothing$. If $\mathrm{EQ} \subseteq r$, then $\mathrm{PO} \subseteq \downarrow r$ and therefore $\downarrow r \in$ $\mathrm{RCA}_{8} \backslash \mathcal{N}$. If EQ $\nsubseteq r$, then $r \cap(\mathrm{TPP} \cup \mathrm{NTPP}) \neq \varnothing$ and $r \cap(\overline{\mathrm{TPP}} \cup \overline{\mathrm{NTPP}}) \neq \varnothing$. Since $r \in \mathrm{RCA}_{8} \backslash \mathcal{N}, \mathrm{PO} \subseteq r$. Thus, PO $\subseteq \downarrow r$ and therefore $\downarrow r \in \mathrm{RCA}_{8} \backslash \mathcal{N}$. Thus, in all cases, $\downarrow r \in \mathrm{RCA}_{8} \backslash \mathcal{N}$. Moreover, we have $\downarrow r \in \mathcal{P}_{8}$ since $\downarrow r \notin V$. Indeed, if EC $\subseteq \downarrow r$, then EC $\subseteq r$ and therefore PO $\subseteq \downarrow r$. By the same argument, we have $\downarrow r \in \mathcal{C}_{8}$. In addition, we have $\downarrow r \in \mathcal{H}_{8} \cap \mathcal{Q}_{8}$ and therefore $\downarrow r \in \mathcal{H}_{8} \cap \mathcal{Q}_{8} \cap \mathcal{C}_{8}$, since if $\mathrm{EQ} \subseteq \downarrow r$ then $\mathrm{EQ} \subseteq r$ and therefore $\mathrm{PO} \cup \mathrm{TPP} \cup \overline{\mathrm{TPP}} \subseteq \downarrow r$.

We end by showing that the subalgebras of the form $\left(\mathcal{H}_{8} \times\left\{\mathcal{H}_{8}, \mathcal{Q}_{8}, \mathcal{C}_{8}\right\}\right)^{\star}$ are algebraically tractable. 
Theorem 1. Let $\mathfrak{R}$ be a topological region space such that $\mathrm{TRCC}_{8}^{\mathrm{d}}$ is a complete sequential formalism.

Subalgebras $\mathcal{S}$ of $\mathrm{TRCC}_{8}^{\mathrm{d}}$ satisfying $S_{2 i-1}=\mathcal{H}_{8}$ and $\mathcal{S}_{2 i} \in\left\{\mathcal{H}_{8}, \mathcal{Q}_{8}, \mathcal{C}_{8}\right\}$ for all $i \in\{1, \ldots, l\}$ are algebraically tractable.

Proof. Let $\mathcal{S}$ be a subset of TRCC ${ }_{8}^{\mathrm{d}}$ satisfying $S_{2 i-1}=\mathcal{H}_{8}$ and $\mathcal{S}_{2 i} \in\left\{\mathcal{H}_{8}, \mathcal{Q}_{8}, \mathcal{C}_{8}\right\}$ for all $i \in\{1, \ldots, l\} . \mathcal{S}$ satisfies the conditions of the first part of the refinement theorem (i.e. $\mathcal{S}$ satisfies the conditions of the first implication ; see the proof of Proposition (4). $\mathcal{S}$ also satisfies the conditions of the second part (i.e. the conditions of the second implication). Indeed, on the one hand, $\mathcal{S}$ is a subclass (since $\mathcal{S}$ is Cartesian and each $\mathcal{S}_{i}$ is a subclass [38]). On the other hand, $\mathcal{S}$ is 户-closed (by Lemma 5 and since $\mathcal{S}$ is a Cartesian subclass). $\mathcal{S}$ is thus algebraically tractable (refinement theorem [7]).

\section{Conclusion}

First, we have focused on $\mathrm{TRCC}_{8}^{\mathrm{n}}$, the qualitative formalism of topological temporal sequences describing the evolution of regions at instants between which there are no intermediary relations (i.e. at time points which characterize all the qualitative changes). We have shown that there is no algebraically tractable Cartesian subalgebra (subclass containing all basic relations) for $\mathrm{TRCC}_{8}^{\mathrm{n}}$ when the length of sequences is longer than 3 . However, we have identified some tractable subclasses. The price of tractability has been to give up the relations containing NTPP not containing TPP and thus to give up the basic relation NTPP.

Then, we have formalized $\mathrm{TRCC}_{8}^{\mathrm{d}}$, the qualitative formalism of topological temporal sequences describing the evolution of regions on a partition of time (i.e. on a contiguous alternation of instants and open intervals). In this context, we have identified large algebraically tractable Cartesian subalgebras.

It is possible to identify other algebraically tractable subclasses for $\mathrm{TRCC}_{8}^{\mathrm{n}}$ and $\operatorname{TRCC}_{8}^{\mathrm{d}}$. The tractability limit of the subclasses of these two formalisms should be precisely determined. In particular, a definitive answer to the question of the existence of polynomial Cartesian subalgebra for $\mathrm{TRCC}_{8}^{\mathrm{n}}$ should be given. Note that the identification of universes ensuring the completeness of $\mathrm{TRCC}_{8}^{\mathrm{n}}$ and of $\mathrm{TRCC}_{8}^{\mathrm{d}}$ remains an open problem, on which we are working.

Concerning the applications, $\operatorname{TRCC}_{8}^{\mathrm{n}}$ and $\mathrm{TRCC}_{8}^{\mathrm{d}}$ can be used to decide if it is possible to go from a topological scenario $S$ to another $S^{\prime}$, with at most $m$ qualitative changes, while satisfying at each instant the constraints of a network $N$ and to determine one of the corresponding intermediate temporal sequences. This problem should be useful for spatial planning. When $S, S^{\prime}$, and $N$ correspond to one of the previous algebraically tractable subclasses (for instance when $S, S^{\prime}$, and $N$ are over $\mathcal{H}_{8}$ ), the problem is polynomial. Otherwise, it is possible that using tractable subclasses still speeds up the resolution of the problem, as in the classic case [40]. Note that $\mathrm{TRCC}_{8}^{\mathrm{d}}$ is more interesting than $\mathrm{TRCC}_{8}^{\mathrm{n}}$ for this problem since it allows to find a more expressive intermediate sequence while having larger tractable subclasses. 


\section{References}

1. Bennett, B., Cohn, A.G., Torrini, P., Hazarika, S.M.: Describing rigid body motions in a qualitative theory of spatial regions (2000)

2. Bennett, B., Cohn, A.G., Wolter, F., Zakharyaschev, M.: Multi-dimensional modal logic as a framework for spatio-temporal reasoning. Applied Intelligence 17(3), 239-251 (2002)

3. Burrieza, A., Muñoz-Velasco, E., Ojeda-Aciego, M.: A pdl approach for qualitative velocity. International Journal of Uncertainty, Fuzziness and Knowledge-Based Systems 19(01), 1126 (2011)

4. Burrieza, A., Ojeda-Aciego, M.: A multimodal logic approach to order of magnitude qualitative reasoning with comparability and negligibility relations. Fundamenta Informaticae 68(1-2), 21-46 (2005)

5. Chen, J., Cohn, A.G., Liu, D., Wang, S., Ouyang, J., Yu, Q.: A survey of qualitative spatial representations. The Knowledge Engineering Review 30(01), 106-136 (2015)

6. Cohen-Solal, Q.: Tractable fragments of temporal sequences of topological information. In: International Conference on Principles and Practice of Constraint Programming. pp. 107125. Springer (2020)

7. Cohen-Solal, Q., Bouzid, M., Niveau, A.: Checking the consistency of combined qualitative constraint networks. In: Proc. of AAAI (2017)

8. Cohen-Solal, Q., Bouzid, M., Niveau, A.: Temporal sequences of qualitative information: Reasoning about the topology of constant-size moving regions. In: Twenty-Sixth International Joint Conference on Artificial Intelligence. pp. 986-992 (2017)

9. Cohn, A.G., Gotts, N.M., Cui, Z., Randell, D.A., Bennett, B., Gooday, J.: Exploiting temporal continuity in qualitative spatial calculi. Spatial and Temporal Reasoning in Geographic Information Systems pp. 5-24 (1998)

10. Cohn, A.G., Hazarika, S.M.: Qualitative spatial representation and reasoning: An overview. Fundamenta informaticae 46(1-2), 1-29 (2001)

11. Davis, E.: Continuous shape transformation and metrics on regions. Fundamenta Informaticae 46(1-2), 31-54 (2001)

12. Dylla, F., Lee, J.H., Mossakowski, T., Schneider, T., Delden, A.V., Ven, J.V.D., Wolter, D.: A survey of qualitative spatial and temporal calculi: algebraic and computational properties. ACM Computing Surveys (CSUR) 50(1), 7 (2017)

13. Dylla, F., Moratz, R.: Exploiting qualitative spatial neighborhoods in the situation calculus. In: Proc. of the International Conference on Spatial Cognition. pp. 304-322. Springer (2004)

14. Dylla, F., Wallgrün, J.O.: Qualitative spatial reasoning with conceptual neighborhoods for agent control. Journal of Intelligent and Robotic Systems 48(1), 55-78 (2007)

15. Egenhofer, M.J.: The family of conceptual neighborhood graphs for region-region relations. In: Proc. of the International Conference on Geographic Information Science. pp. 42-55. Springer (2010)

16. Egenhofer, M.J.: Qualitative spatial-relation reasoning for design. In: Studying Visual and Spatial Reasoning for Design Creativity, pp. 153-175. Springer (2015)

17. Freksa, C.: Conceptual neighborhood and its role in temporal and spatial reasoning. In: Proc. of the IMACS Workshop on Decision Support Systems and Qualitative Reasoning. pp. 181187 (1991)

18. Freksa, C.: Temporal reasoning based on semi-intervals. Artif. Intell. 54(1-2), 199-227 (1992)

19. Gabelaia, D., Kontchakov, R., Kurucz, A., Wolter, F., Zakharyaschev, M.: On the computational complexity of spatio-temporal logics. In: FLAIRS Conference. pp. 460-464 (2003)

20. Galton, A.: Towards an integrated logic of space, time, and motion. In: Proc. of IJCAI. pp. 1550-1555 (1993) 
21. Galton, A.: Qualitative spatial change. Oxford University Press (2000)

22. Galton, A.: Dominance diagrams: A tool for qualitative reasoning about continuous systems. Fundamenta Informaticae 46(1-2), 55-70 (2001)

23. Gerevini, A., Nebel, B.: Qualitative spatio-temporal reasoning with RCC-8 and Allen's interval calculus: Computational complexity. In: Proc. of ECAI. pp. 312-316 (2002)

24. Hazarika, S.M., Cohn, A.G.: Qualitative spatio-temporal continuity. In: Proc. of the International Conference on Spatial Information Theory. pp. 92-107. Springer (2001)

25. Hazarika, S.M.: Qualitative spatial change: space-time histories and continuity. Ph.D. thesis, University of Leeds (2005)

26. Kowalski, R., Sergot, M.: A logic-based calculus of events. In: Foundations of knowledge base management, pp. 23-55. Springer (1989)

27. Kurata, Y., Egenhofer, M.J.: The 9+-intersection for topological relations between a directed line segment and a region.

28. Landsiedel, C., Rieser, V., Walter, M., Wollherr, D.: A review of spatial reasoning and interaction for real-world robotics. Advanced Robotics 31(5), 222-242 (2017)

29. Li, S., Ying, M.: Region connection calculus: Its models and composition table. Artificial Intelligence 145(1-2), 121-146 (2003)

30. Ligozat, G.: Towards a general characterization of conceptual neighborhoods in temporal and spatial reasoning. In: AAAI 1994 TheTwelfth National Conference on Artificial Intelligence (1994)

31. Ligozat, G.: Qualitative spatial and temporal reasoning. John Wiley \& Sons (2013)

32. Mansouri, M., Pecora, F.: A robot sets a table: a case for hybrid reasoning with different types of knowledge. Journal of Experimental \& Theoretical Artificial Intelligence 28(5), 801-821 (2016)

33. Muller, P.: Topological spatio-temporal reasoning and representation. Computational Intelligence 18(3), 420-450 (2002)

34. Ragni, M., Wölfl, S.: Temporalizing cardinal directions: From constraint satisfaction to planning. In: Proc. of KR. pp. 472-480 (2006)

35. Ragni, M., Wölfl, S.: Reasoning about topological and positional information in dynamic settings. In: Proc. of the FLAIRS Conference. pp. 606-611 (2008)

36. Randell, D.A., Cui, Z., Cohn, A.G.: A spatial logic based on regions and connection. In: Proc. of KR. pp. 165-176 (1992)

37. Reis, R.M., Egenhofer, M.J., Matos, J.L.: Conceptual neighborhoods of topological relations between lines. In: Headway in Spatial Data Handling, pp. 557-574. Springer (2008)

38. Renz, J.: Maximal tractable fragments of the region connection calculus: A complete analysis. In: Proc. of IJCAI. pp. 448-455 (1999)

39. Renz, J., Ligozat, G.: Weak composition for qualitative spatial and temporal reasoning. In: Proc. of CP, pp. 534-548. Springer (2005)

40. Renz, J., Nebel, B.: Efficient methods for qualitative spatial reasoning. Journal of Artificial Intelligence Research 15, 289-318 (2001)

41. Santos, M.Y., Moreira, A.: Conceptual neighborhood graphs for topological spatial relations. In: Proc. of the World Congress on Engineering. vol. 1, pp. 12-18 (2009)

42. Sioutis, M., Alirezaie, M., Renoux, J., Loutfi, A.: Towards a synergy of qualitative spatiotemporal reasoning and smart environments for assisting the elderly at home. In: IJCAI Workshop on Qualitative Reasoning. pp. 901-907 (2017)

43. Sioutis, M., Condotta, J.F., Salhi, Y., Mazure, B.: A qualitative spatio-temporal framework based on point algebra. In: Proc. of AIMSA. pp. 117-128. Springer (2014)

44. Sioutis, M., Condotta, J.F., Salhi, Y., Mazure, B.: Generalized qualitative spatio-temporal reasoning: Complexity and tableau method. In: International Conference on Automated Reasoning with Analytic Tableaux and Related Methods. pp. 54-69. Springer (2015) 
45. Sioutis, M., Condotta, J.F., Salhi, Y., Mazure, B., Randell, D.A.: Ordering spatio-temporal sequences to meet transition constraints: Complexity and framework. In: Proc. of AI Applications and Innovations. pp. 130-150. Springer (2015)

46. Van Benthem, J.: The logic of time: a model-theoretic investigation into the varieties of temporal ontology and temporal discourse, vol. 156. Springer Science \& Business Media (2013)

47. Van de Weghe, N., De Maeyer, P.: Conceptual neighbourhood diagrams for representing moving objects. In: Proc. of the International Conference on Conceptual Modeling. pp. 228238. Springer (2005)

48. Westphal, M., Dornhege, C., Wölfl, S., Gissler, M., Nebel, B.: Guiding the generation of manipulation plans by qualitative spatial reasoning. Spatial Cognition \& Computation 11(1), 75-102 (2011)

49. Westphal, M., Hué, J., Wölfl, S., Nebel, B.: Transition constraints: A study on the computational complexity of qualitative change. In: Proc. of IJCAI. pp. 1169-1175 (2013)

50. Westphal, M., Wölfl, S.: Qualitative csp, finite csp, and sat: Comparing methods for qualitative constraint-based reasoning. In: Twenty-First International Joint Conference on Artificial Intelligence (2009)

51. Wolter, F., Zakharyaschev, M.: Spatio-temporal representation and reasoning based on rcc-8. In: KR. pp. 3-14 (2000)

52. Wolter, F., Zakharyaschev, M.: Qualitative spatio-temporal representation and reasoning: a computational perspective. Exploring Artificial Intelligence in the New Millenium pp. 175216 (2002)

53. Wu, J., Claramunt, C., Deng, M.: Towards a qualitative representation of movement. In: Proc. of the International Conference on Conceptual Modeling. pp. 191-200. Springer (2014)

54. Zimmermann, K., Freksa, C.: Enhancing spatial reasoning by the concept of motion. Prospects for Artificial Intelligence pp. 140-147 (1993) 